Purdue University

Purdue e-Pubs

CTRC Research Publications

Cooling Technologies Research Center

$8-21-2008$

\title{
Review and Comparative Analysis of Studies on Saturated Flow Boiling in Small Channels,
}

Stefan Bertsch

Eckhard A. Groll

Purdue University - Main Campus, groll@purdue.edu

S V. Garimella

Purdue University, sureshg@purdue.edu

Follow this and additional works at: http://docs.lib.purdue.edu/coolingpubs

Bertsch, Stefan; Groll, Eckhard A.; and Garimella, S V., "Review and Comparative Analysis of Studies on Saturated Flow Boiling in Small Channels," (2008). CTRC Research Publications. Paper 91.

http://dx.doi.org/10.1080/15567260802317357

This document has been made available through Purdue e-Pubs, a service of the Purdue University Libraries. Please contact epubs@purdue.edu for additional information. 


\title{
Review and Comparative Analysis of Studies on Saturated Flow Boiling in Small Channels
}

\author{
Stefan S Bertsch ${ }^{1,2}$, Eckhard A. Groll ${ }^{1,2}$, Suresh V. Garimella ${ }^{2, *}$ \\ ${ }^{1}$ Ray W. Herrick Laboratories and ${ }^{2}$ Cooling Technologies Research Center \\ School of Mechanical Engineering, Purdue University \\ 585 Purdue Mall, West Lafayette, IN 47907-2088 USA
}

\begin{abstract}
Recent studies relating to saturated flow boiling in minichannels and microchannels are reviewed, with a focus on the functional dependence of the heat transfer characteristics on thermodynamic vapor quality. While there is general agreement in the literature that the heat transfer coefficient increases with increases in heat flux, conflicting trends have been reported on the influence of vapor quality. A compilation and analysis of the results from recent investigations in the literature on flow boiling in small channels is presented. Further, correlations for flow boiling proposed in these studies are quantitatively assessed by comparing them against ten independent data sets from the published literature covering a range of hydraulic diameters from 0.16 to $2.01 \mathrm{~mm}$. Recommended topics for future research in this field are also identified.
\end{abstract}

Keywords: microchannel, minichannel, flow boiling, heat transfer, boiling, vapor quality

* To whom correspondence should be addressed: sureshg@ purdue.edu, 765-494-5621 


\section{Introduction}

The recent surge in the number of publications on flow boiling heat transfer in minichannels and microchannels has been motivated to a large extent by the potential for high-flux heat removal in applications such as microelectronic devices [1-4]. Flow boiling offers the capability of addressing the thermal management challenges resulting from the steady increase in gate density of electronic chips over the last few decades, while at the same time minimizing temperature gradients across the chip [5].

Several reviews related to minichannel and microchannel heat transfer, pressure drop, critical heat flux, flow instabilities and flow patterns are available in the literature. While some reviews have covered a range of topics such as the comparison of different approaches for high-heat-flux cooling [6,7] and the evolution of high-flux heat transfer approaches $[8,9]$, others have focused on specific topics such as flow instabilities, flow patterns, CHF [2, 10-14], and pressure drop in small tubes and channels [1]. Heat transfer during flow boiling in microchannels has been reviewed [3, 15-17], and the applicability of existing correlations assessed [18-20].

Saturated flow boiling in microchannels and the associated heat transfer mechanisms are not as well understood as the fluid dynamics and heat transfer characteristics of single-phase flow [8]. In particular, as will be evident from the detailed review of the literature presented here, very limited attention has been directed towards details of the influence of thermodynamic vapor quality on the heat transfer coefficient in microchannels. Knowledge of the flow boiling heat transfer coefficient as a function of vapor quality is essential in applications such as vapor compression-based refrigeration, which has been shown to be an effective means for cooling electronics [21-23]. All of 
the refrigerant liquid stream goes through a change of phase to the vapor state in such systems. Results from the subcooled boiling often encountered in liquid loops, on the other hand, only consider a very limited quality range, and may not be applied to the design of vapor compression refrigeration systems. Even from the few studies of microchannel flow boiling that considered a wide range of vapor quality, conflicting results were reported. Some of these studies observed behavior similar to that in conventional-sized channels, with the heat transfer coefficient increasing as the vapor quality increases $[24,25]$ until dryout occurs. Early work in this area reported the heat transfer coefficient to be independent of vapor quality $[26,27]$. On the other hand, more recent studies found the heat transfer to peak at low vapor qualities and drop significantly towards higher thermodynamic qualities [28-31].

The objective of the present review of flow boiling in microchannels is to identify the trends in the literature on which there is broad agreement. Some of the governing parameters have also been reported to have conflicting effects in different studies, and these conflicts are discussed. The first part of the review summarizes the key findings from recent studies on flow boiling in minichannels and microchannels. In the second part, 25 published heat transfer correlations are compared and assessed for their ability to predict experimental measurements. For the measurements, 1847 data points are compiled from ten different published studies which report flow boiling heat transfer measurements in channels of hydraulic diameter less than $2 \mathrm{~mm}$. The aim of the present study is to identify the applicability of correlations developed both for conventional-sized and microscale channels in predicting the heat transfer coefficient in small channels. Correlations that show the best agreement with the compiled experimental database are 
identified. The review also highlights topics in microchannel flow boiling on which additional research is needed.

\section{Review of the Literature}

The review presented here considers saturated flow boiling studies, and is intended as a follow-up to the extensive literature reviews presented in $[1,8]$ where the focus was on single-phase flow and heat transfer. Studies from the past decade on flow boiling in microchannels, minichannels, and small tubes are presented in chronological order in Table 1, in which the influence of heat flux, mass flux, flow patterns, and hydraulic diameter on heat transfer, pressure drop and flow characteristics are highlighted. The studies are categorized according to whether the main focus is on heat transfer coefficient, pressure drop, critical heat flux, or flow patterns and flow instabilities. For each study, the operating conditions and physical dimensions are listed together with a summary of the main observations and conclusions of the study. The work included in the table considers both measurements as well as simulations and modeling.

While the studies summarized in Table 1 focus on all aspects of flow boiling in microchannels, the primary focus in the discussion in this section is on the variation of heat transfer coefficient with vapor quality.

Figure 1 reproduces experimental results for the heat transfer coefficient as a function of thermodynamic quality from six different studies [24, 28-30, 32, 33] for channels with hydraulic diameters in the range of 1 to $2 \mathrm{~mm}$ or less. The operating conditions in all six studies are comparable with respect to hydraulic diameter, heat flux, and mass flux, allowing for a direct comparison of the results. 
Bao et al. [32] found little or no influence of vapor quality on the heat transfer coefficient as shown in Figure 1 (a). Such a trend was also noted in other studies on small channels [26, 27, 34]. In contrast, Yan and Lin [28] found a strong dependence of the heat transfer coefficient on vapor quality, mass flux, and saturation temperature within the investigated operating range. Figure 1 (b) shows the heat transfer coefficient as a function of vapor quality at different saturation temperatures and imposed heat fluxes from that study [28]. An array of circular copper minichannels with an inside diameter of $2 \mathrm{~mm}$ was tested with the fluid R134a. Heat transfer results in the small tubes showed varying trends under different operating conditions but were generally $30 \%$ to $80 \%$ higher for the minichannels when compared to conventional-sized tubes. Figure 1(c) shows the heat transfer coefficient as a function of vapor quality for different heat fluxes as determined by Lin et al. [29], who found quite similar results for flow boiling of $\mathrm{R} 141 \mathrm{~b}$ in a circular tube of diameter $1.1 \mathrm{~mm}$. Mass fluxes in the range of 300 to $2000 \mathrm{~kg}$ $\mathrm{m}^{-2} \mathrm{~s}^{-1}$ and vapor qualities of -0.1 (indicating a subcooled liquid state) to 1.0 were investigated.

Bertsch et al. [30] also found a strong dependence of the heat transfer coefficient on heat flux and thermodynamic vapor quality. The heat transfer coefficient with R134a was found to increase with heat flux (Figure 1 (d)) and to peak at a vapor quality of approximately 0.2 in measurements obtained in a copper test piece with 17 parallel microchannels of hydraulic diameter $1.09 \mathrm{~mm}$. No significant influence of the saturation temperature on the heat transfer coefficient was discerned. The flow patterns observed were bubbly flow (for subcooled and very low vapor qualities), slug flow (up to vapor qualities of approximately 0.2 ), and then intermittent annular flow thereafter. Out-of- 
phase flow instabilities (opposing flow directions in adjacent channels) with high oscillation frequencies occurred especially at low mass flux and high heat flux conditions.

Yet another study that showed a dependence of heat transfer coefficient on vapor quality was that of Yun et al. [33], who considered flow boiling of refrigerant R410A in an array of rectangular microchannels with hydraulic diameters of $1.36 \mathrm{~mm}$ and $1.44 \mathrm{~mm}$. The results (Figure 1 (e)) showed a peak in heat transfer coefficient between a vapor quality of 0.4 and 0.6. The significant deviation observed between their measurements and predictions obtained from conventional correlations for single tubes was attributed to the effect of channel diameter and the difference in behavior between single and multiple parallel channels. Saturation temperature was again found to have relatively little influence on the heat transfer coefficient. Agostini et al. [31] also found a sharp peak in heat transfer coefficient at low vapor qualities with a steady decrease towards higher vapor qualities. The measurements in [31] were carried out in two aluminum microchannel heat exchangers with hydraulic diameters of $2.01 \mathrm{~mm}$ and $0.77 \mathrm{~mm}$. The critical vapor quality (vapor quality at which the heat transfer coefficient starts to rapidly decrease) was found to be 0.4 for the larger channels and 0.2 for the small channels.

A detailed investigation of heat transfer coefficient versus vapor quality with respect to tube size was also conducted by Saitoh et al. [24]. Measurements were conducted for vapor qualities of 0.2 and higher, and results for a tube of $1.12 \mathrm{~mm}$ diameter are shown in Figure 1 (f). As in many of the other studies discussed, the heat transfer coefficient first increased and then sharply decreased towards higher vapor qualities. The peak in heat transfer coefficient appears to move towards lower vapor qualities as the tube diameter 
was decreased: for tube diameters of $3.1 \mathrm{~mm}, 1.12 \mathrm{~mm}$, and $0.51 \mathrm{~mm}$, the peaks in heat transfer coefficient occur at thermodynamic vapor qualities of $0.9,0.85$, and 0.5 , respectively. According to the authors, the contribution of forced convection to the total heat transfer decreases with decreasing tube diameter, resulting in a dominant nucleate boiling heat transfer regime. This increased importance of nucleate boiling in small channels compared to conventional-sized channels has been reported in a large number of investigations.

A number of other studies in the literature have reported conflicting trends. Lee and Lee [25] performed flow boiling experiments with refrigerant R113 using rectangular low-aspect-ratio channels. The plate heat exchanger used had channel heights of 0.4 to $2.0 \mathrm{~mm}$ with a fixed channel width of $20 \mathrm{~mm}$. The heat transfer coefficient increased with mass flux and quality; however, the effect of heat flux appeared to be minor. Whereas the heat transfer coefficient in this case increased with vapor quality, Steinke and Kandlikar $[16,35]$ found the exact opposite trend in their measurements on six parallel rectangular microchannels of hydraulic diameter $207 \mu \mathrm{m}$. The authors $[16,35]$ also found the full range of flow patterns seen in conventional-sized channels: nucleate boiling, bubbly flow, slug flow, annular flow, annular flow with nucleation in the thin film, churn flow, and dry-out. In addition, flow reversal and flow instabilities were observed in the microchannel array under certain conditions.

It is evident from this discussion and from Table 1 that a large number of studies on two-phase flow and convective boiling in small channels are available in the literature. Various combinations of channel size, channel geometry, and substrate material have been considered along with a wide selection of working fluids. However, a detailed 
understanding of the mechanisms of convective flow boiling in microchannels, leading to the ability to predict heat transfer coefficient under a wide range of conditions for design purposes, continues to be elusive. There is broad agreement on the following trends: the heat transfer coefficient increases with heat flux; mass flux has only a modest influence on heat transfer coefficient, with the heat transfer coefficient remaining unaffected or increasing somewhat with an increase in mass flux; and nucleate boiling plays an influential role in microchannel flow boiling, especially at the low mass fluxes. In addition, the effect of saturation temperature on heat transfer coefficient is relatively small. The reported trends of variation of the heat transfer coefficient with vapor quality, on the other hand, have been contradictory, with different papers observing opposing trends for similar operating conditions. This is one area in which more investigation is needed before firm conclusions can be drawn.

\section{Prediction of Heat Transfer Coefficient}

A large number of heat transfer correlations that have been proposed in various studies for prediction of the heat transfer coefficient in flow boiling in tubes and channels are examined here. A majority of the correlations were developed for conventional-sized channels, while some were specifically developed for minichannels and microchannels. Besides flow boiling heat transfer correlations, two widely used pool boiling correlations are also included in the comparison. Table 2 provides a summary of the conditions and ranges of applicability of the correlations considered in this comparison.

It may be noted that several typographical errors, missing definitions and errata were discovered in a number of the original publications. Some of these were resolved via 
personal communication with the authors of the original papers. Also, to ensure that the correlations were interpreted correctly, their predictions were compared to the original data sets published in the paper, whenever possible. To aid in the ease of use of these correlations, and to avoid further difficulties in interpreting correlations from some of the original sources, an appendix is provided ${ }^{\dagger}$ which contains a complete listing of the correlations, definitions, geometries, and the range of applicability for each of the papers included in the present comparison.

The required fluid properties were calculated using Engineering Equation Solver (EES) [36] which evaluates properties as a function of pressure and temperature or vapor quality. Surface tension of a few fluids not contained in EES was correlated with a polynomial fit to data obtained from Refprop7 [37] with a deviation of less than $0.15 \%$ within the operating range. In addition, all properties of the fluid FC-77 were obtained as a curvefit to data from the manufacturer [38] with a deviation of less than $0.2 \%$.

Figure 2 shows the predicted heat transfer coefficients from each correlation as a function of vapor quality as applied to the flow boiling of refrigerant R134a in a copper channel of diameter $1.0 \mathrm{~mm}$, with a mass flux of $500 \mathrm{~kg} \mathrm{~m}^{-2} \mathrm{~s}^{-1}$, a heat flux of $10 \mathrm{~W} \mathrm{~cm}^{-2}$, and a saturation temperature of $20^{\circ} \mathrm{C}$ which corresponds to a pressure of $572 \mathrm{kPa}$. The flow is assumed fully developed and the wall surface temperature needed for property calculations is assumed to be $25^{\circ} \mathrm{C}$. The thermodynamic vapor quality $\mathrm{x}$ is chosen as the independent variable and ranges from 0.01 to 0.99 for most equations. For correlations which recommend that predictions be restricted to a certain vapor quality range, the data are only plotted in the appropriate range. The comparison serves to illustrate the

\footnotetext{
$\dagger$ This appendix has been provided as a separate document to the Editor, with the understanding that it will either be included in the printed journal issue, or provided as supplemental information on the journal website.
} 
predicted trends from each correlation of the variation of heat transfer coefficient with vapor quality. Figure 2 (a) shows the results of the correlations for conventional-sized channels, while Figure 2 (b) shows the results for small channels. The majority of the calculated heat transfer coefficients in both plots cluster around a value of $10,000 \mathrm{~W} \mathrm{~m}^{-2}$

$\mathrm{K}^{-1}$, which is broadly consistent with measurement data for refrigerant $\mathrm{R} 134 \mathrm{a}[24,28,30$,

31] in a similar operating range. Whereas most of the correlations developed for conventional-sized channels show little dependence of the heat transfer coefficient on vapor quality, most of the small-channel correlations show a decrease in heat transfer coefficient with an increase in thermodynamic vapor quality with the exception of [25, 68]. These predicted trends of variation with vapor quality are compared to those from experimental data sets in the following section.

\section{Quantitative Assessment of Correlations against Experimental Results}

A database was assembled from ten different published studies with a total of 1847 measured heat transfer data points, and was used for comparing the predictions from the heat transfer correlations discussed above against experimental results. Table 3 describes the data sets extracted by digitizing plots presented in the literature as well as some original data sets acquired in the authors' group, along with a listing of the range of the most important investigated parameters. Criteria by which the data sets were selected include the availability of all the necessary information regarding the tests (mass flux, heat flux, vapor quality, and saturation temperature) along with the complete geometry and fluid information. No data set was used that did not include all of this information. The only parameter that was estimated from fluid temperature, heat flux, and heat transfer coefficient was the wall temperature, if it was not provided in a specific dataset. 
The maximum hydraulic diameter considered for the channels in this comparison is 2 $\mathrm{mm}$. Since the main focus of the comparison is the prediction of the heat transfer coefficient as a function of vapor quality, only data sets that span a large range of thermodynamic vapor quality are included. The overall database spans eight different fluids, which are mostly either refrigerants or wetting dielectric fluids, and four substrate materials including copper, silicon, stainless steel, and aluminum. The database covers a wide range of heat fluxes, mass fluxes, and saturation temperatures, and includes data obtained with single channels as well as multiple parallel channels.

There are a variety of complications in the extraction of heat transfer coefficient with a given measurement apparatus, including the consideration of heat losses and the required solution of the conjugate problem for the case of a spatially varying wall temperature. Also, it is difficult to precisely determine the fluid temperature, due to variations in the pressure (and saturation temperature) along the flow channel. A common measurement technique in the literature has been to use direct electrical heating for thin-walled tubes $[29,31,33,82]$. In this case, measurement of the wall temperature is challenging due to the spatially varying temperature. A slight modification of this technique for silicon microchannels is to use localized heaters and diodes to measure the temperature $[69,74]$. This technique allows for the spatial variation of temperature to be measured. Temperature measurements with an extrapolation approach using a set of thermocouples along the heat flow direction in a heated block can also yield surface temperatures $[30,77]$; however, an excellent heat balance and characterization of heat losses is crucial to the success of this approach. Due to the small channel size, in situ pressure measurements are difficult and the determination of the fluid temperature (if not 
directly measured) at any given point must rely on assumptions regarding the pressure variation along the channel. Therefore, uncertainties in the measured heat transfer coefficients are usually in the range of 10 to $15 \%$.

The measured heat transfer coefficients from the database were compared to predictions obtained for the same conditions from the correlations introduced earlier. The accuracy of the predictions was assessed using the ratio of predicted to measured heat transfer coefficient:

$$
\Phi=\frac{\mathrm{h}_{\text {calc }}}{\mathrm{h}_{\text {meas }}}
$$

The results for twelve correlations are shown in Figure 3, where $\Phi$ is plotted versus the vapor quality on a logarithmic axis in order to accommodate the range of values; all panels in the figure are drawn using the same scale to facilitate visual comparison. Of the investigated correlations listed in Table 2, the most commonly used and those with the lowest deviations are chosen for inclusion in this figure. Different symbols are used to represent different data sets. The line $\Phi=1$ representing a perfect match between prediction and experiment is shown; in addition, dashed lines are included at $\pm 50 \%$ of this line (i.e., at $\Phi=0.5$ and 1.5). It is noted that several of the correlations are projected beyond their suggested vapor quality range, so as to establish how the correlations extrapolate over the entire quality range.

Considering the logarithmic scale of the ratio $\Phi$, the deviation between measurements and predictions is substantial over the entire vapor quality range, and not just at high thermodynamic qualities as would be indicated from the suggested range of applicability for most equations. Also, the deviations are not attributable to a specific fluid or a specific geometry. 
Overall, the Cooper [137] correlation shows the lowest deviation between predictions and measurements for the database used in the comparisons of this study. This is remarkable in view of the fact that this correlation was developed specifically for pool boiling and does not contain any effects of mass flux and vapor quality. Interestingly, the only other pool boiling correlation - that of Gorenflo [138] - also results in smaller deviations from the experimental database than most of the flow boiling correlations. The good agreement between the predictions using the pool boiling correlations and the measurements could indicate a dominance of the nucleate boiling heat transfer regime in microchannels. Many studies have arrived at a similar conclusion with regard to the predominance of the nucleate boiling regime in microchannel flow boiling $[26,27,35$, $40,45,78,53,69,73,79,134]$.

It is also interesting to note that predictions obtained with correlations developed specifically for small channels fared no better than those for conventional-sized tubes in terms of accuracy of the predictions. The agreement seems to be most strongly influenced by the size of the data set on which a correlation was based. Especially for some of the newer correlations, the small data sets considered might explain their lack of applicability beyond their often very narrow range of parameters.

Figures 4 and 5 provide plots similar to that in Figure 3 . The difference is that the ratio of predicted to measured heat transfer coefficient is now plotted as a function of heat flux and mass flux, respectively. The heat flux plotted is based on the heated wetted area of the channel (and not the base area of the heated chip as has been used in some of the published literature). In general, the correlations appear to show better agreement with experiments at the higher heat fluxes and mass fluxes. The effect of heat flux is 
captured better by the correlations (deviating in some cases by a constant factor). The effect of mass flux, on the other hand, is poorly represented by most correlations. A clearer understanding of the underlying physics of microchannel flow boiling obtained from carefully designed experiments and simulations is necessary to achieve better predictions through physics-based models.

In addition to the graphical comparison in Figures 3, 4 and 5 of the predictions obtained with twelve of the correlations considered, several characteristic parameters were calculated to provide a quantitative comparison of all correlations listed in Table 2. The mean relative error (MRE) and the mean absolute error (MAE) were calculated as defined below:

$$
\begin{aligned}
& \mathrm{MRE}=\frac{1}{\mathrm{~N}} \sum_{\mathrm{N}} \frac{\mathrm{h}_{\text {calc }}-\mathrm{h}_{\text {meas }}}{\mathrm{h}_{\text {meas }}} \\
& \mathrm{MAE}=\frac{1}{\mathrm{~N}} \sum_{\mathrm{N}} \frac{\left\|\mathrm{h}_{\text {calc }}-\mathrm{h}_{\text {meas }}\right\|}{\mathrm{h}_{\text {meas }}}
\end{aligned}
$$

In addition, the number of predicted heat transfer coefficients that fall within $\pm 30 \%$ of the measured data is determined. These three measures of the goodness of the comparisons are presented in Table 4 for all the correlations considered, with respect to the ten experimental data sets used in the comparison (as listed in Table 3). In addition, Table 4 contains the average value calculated as the mean of the ten respective values. In computing values in this table, the upper limit of the thermodynamic vapor quality range was restricted to $70 \%$ for the calculation of the three goodness parameters. Weighting the average by number of data sets and not the number of total data points ensured that the comparison was not skewed due to different sizes of data sets over specific operating ranges. Mean absolute error (MAE) values of less than $30 \%$ are highlighted in bold font 
for ease of identification. In addition, if more than half of the data points were predicted to within $\pm 30 \%$, the number is highlighted in bold as well.

The overall best prediction - identified as that which provides the lowest MAE and the largest percentage of data predicted to within $\pm 30 \%$ of the measurements - is achieved by the Cooper [137] correlation. This confirms the conclusions already drawn above from Figures 3, 4 and 5. Other correlations that show reasonable agreements with the given database include those of Haynes and Fletcher [134], Gorenflo (a pool boiling correlation) [138], Liu and Winterton [132], Thome et al. [62, 63] and Tran et al. [27]. Both Haynes and Fletcher and Liu and Winterton used superposition approaches and rely on the pool boiling equations of Cooper and Gorenflo, which might explain their success.

Of the many investigated variations of the Chen correlation, the original version [125] with the modifications of Edelstein et al. [124] and of Bennett and Chen [126] and Bennett et al. [127] show reasonable and comparable results. A few of the more recent modifications of the Chen correlation improve upon the agreement with the experimental database, with Saitoh et al. [82] and Liu and Winterton [132] showing the lowest MAE of such correlations. In addition, of the two modifications of the Kandlikar correlation, the newer correlation [135] designed for small channels provides marginally (within 1\%) better results compared to the original version [130].

In very recent work, a modified Chen-type heat transfer correlation [139] has been developed by the authors. This correlation modifies the successful Cooper correlation to take bubble confinement in small channels into account. This physically sound yet simple correlation was validated against a database compiled from 14 studies and showed a mean absolute error of $28 \%$ compared to the $39 \%$ obtained with the Cooper correlation. 
In summary, even those correlations that are identified in this review to be the best available are not very accurate. Additional research into the mechanisms of flow boiling in small channels is necessary. Models based on the physics of the boiling process and the appropriate flow patterns and flow regime maps are likely to yield better to extrapolation beyond the boundaries of the operating ranges (mass flux, heat flux, vapor quality, and hydraulic diameter) relative to purely empirical curvefits. So far, few such models based on the flow regimes have been published. One of these, due to Thome et al. [62, 63], was fairly successful over a broad parameter range, but its main drawback lies in its assumption of a single regime (elongated bubble flow), which conflicts with the observation of multiple flow regimes by several researchers $[2,30,35,74]$ as well as the trends of several heat transfer measurements $[14,16,19]$. Similarly Qu and Mudawar [57] proposed a regime-based model, which assumed only an annular flow regime.

Well-characterized experiments that investigate flow regimes and instabilities over a broad range of parameters (fluids, substrates, and geometries) will help to achieve a better understanding of the underlying heat transfer processes on which more realistic models can be based.

\section{Conclusions}

A review of the literature on flow boiling in small channels is presented, with a focus on the variation of heat transfer coefficient with thermodynamic vapor quality.

Predictions from 25 heat transfer correlations in the literature are compared to 1847 measurements from ten independent sources. The hydraulic diameter of the tubes and 
channels considered in all the experiments in the database is $2 \mathrm{~mm}$ or less. The following conclusions are drawn based on the review and comparative analysis:

- Predictions of the heat transfer coefficient from the available correlations have only achieved partial success. The best predictions still show a mean absolute error (MAE) of $40 \%$ relative to the large experimental database, and predict less than half of the measured data to within a deviation of $\pm 30 \%$. Predictions from twelve out of the 25 correlations exceed errors of $100 \%$.

- Correlations that have been developed especially for minichannels and microchannels essentially show no improvements over those developed earlier for conventional-sized channels.

- Not surprisingly, correlations that were proposed based on a curvefit to a single data set do not extrapolate well beyond the very narrow parameter ranges of the original tests.

- The correlation that showed the best agreement with the experimental measurements database is the pool boiling correlation of Cooper [137]. None of the correlations developed for flow boiling offered an improved prediction. The Cooper correlation underpredicts the heat transfer coefficient at low thermodynamic vapor qualities and slightly overpredicts at high qualities.

- From the quantitative comparison presented in this work, nucleate boiling appears to dominate the heat transfer process in microchannels. Both pool boiling equations investigated $[137,138]$ result in better predictions than all of the flow boiling equations; one flow boiling correlation led to comparable results [134]. 
Many studies in the literature have also drawn similar conclusions about the predominance of nucleate boiling in microchannels.

There is a clear need for physics-based models of microchannel flow boiling to be developed. Such models should be validated against large databases of experimental measurements covering a wide range of parameters. Few correlations in the current literature are based on the prevalent flow regimes in microchannels; even those that are developed in this manner have usually considered a single flow regime. It is important that flow regime maps be first developed based on simultaneous high-speed visualization and local heat transfer and pressure drop measurements in carefully characterized experimental setups. Such a broad database would then allow the development and validation of models that are informed by the flow regimes to be expected for a given set of parameters.

\section{Acknowledgements}

The authors acknowledge financial support for this work from members of the Cooling Technologies Research Center, a National Science Foundation Industry / University Cooperative Research Center at Purdue University.

\section{Nomenclature}

CHF critical heat flux

D diameter, $\mathrm{m}$

G mass flux, $\mathrm{kg} \mathrm{m}^{-2} \mathrm{~s}^{-1}$

$\mathrm{h} \quad$ heat transfer coefficient, $\mathrm{W} \mathrm{m}^{-2} \mathrm{~K}^{-1}$

$\mathrm{H} \quad$ channel height, $\mathrm{m}$ 


\begin{tabular}{|c|c|}
\hline $\mathrm{m}$ & mass flow rate, $\mathrm{kg} \mathrm{s}^{-1}$ \\
\hline MAE & mean absolute error, $\%$ \\
\hline MRE & mean relative error, $\%$ \\
\hline $\mathrm{N}$ & number of data points \\
\hline ONB & Onset of nucleate boiling \\
\hline $\mathrm{p}$ & pressure, $\mathrm{Pa}$ \\
\hline q" & heat flux, $\mathrm{W} \mathrm{m} \mathrm{m}^{-2}$ \\
\hline Q & heat rate, $\mathrm{W}$ \\
\hline $\operatorname{Re}$ & Reynolds number \\
\hline RMS & root mean square \\
\hline $\mathrm{T}$ & temperature, ${ }^{\circ} \mathrm{C}$ \\
\hline $\mathrm{V}$ & velocity, $\mathrm{m} \mathrm{s}^{-1}$ \\
\hline $\mathrm{W}$ & channel width, m \\
\hline $\mathrm{x}$ & thermodynamic quality \\
\hline
\end{tabular}

Greek:

$\Phi \quad$ ratio of calculated to measured heat transfer coefficient

\begin{tabular}{ll}
\multicolumn{2}{l}{ Subscripts: } \\
el & electric \\
$\mathrm{h}$ & hydraulic \\
in & inlet \\
max & maximum \\
meas & measured \\
calc & calculated \\
out & outlet \\
sat & saturated
\end{tabular}


Table 1. Summary of studies in the literature related to flow boiling in microchannels, categorized according to the primary focus of each study.

\begin{tabular}{|c|c|c|c|}
\hline Reference & $\begin{array}{l}\text { Fluid/Substrate, } \\
\text { Geometry, } D_{h}[\mathrm{~mm}], \mathbf{N}\end{array}$ & 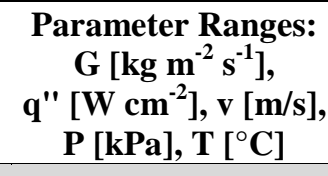 & Remarks \\
\hline \multicolumn{4}{|c|}{ Heat Transfer Coefficient } \\
\hline $\begin{array}{l}\text { Lazarek and Black } \\
\text { (1982) [26] }\end{array}$ & $\begin{array}{l}\text { R113 in copper } \\
\text { circular } \\
\mathrm{D}=3.1\end{array}$ & $\begin{array}{l}\mathrm{G}=125-750 \\
\mathrm{Re}=860-5500 \\
\mathrm{q}^{\prime \prime}=0-40 \\
\mathrm{x}=0-0.8\end{array}$ & $\begin{array}{l}\text { Measurement of local heat transfer, pressure drop and CHF: } \\
\text { h is relatively independent of } \mathrm{x} \text {, and a function of } \mathrm{Nu}, \mathrm{Re} \text {, and Bo } \\
\text { Nucleate boiling dominates the heat transfer }\end{array}$ \\
\hline $\begin{array}{l}\text { Peng and Wang } \\
\text { (1993) [39] }\end{array}$ & $\begin{array}{l}\text { water in stainless steel } \\
\text { rectangular } \\
\mathrm{D}_{\mathrm{h}}=0.646\end{array}$ & $\begin{array}{l}\operatorname{Re}=1000-6000 \\
q^{\prime \prime}=1-100 \\
v=1.5-4\end{array}$ & $\begin{array}{l}\text { Experiments on subcooled boiling of water: } \\
\text { Nucleate boiling is intensified while wall superheat for flow boiling decreases in } \\
\text { microchannels relative to conventional-sized channels for the same wall heat flux } \\
\text { No partial nucleate boiling is observed in microchannels }\end{array}$ \\
\hline $\begin{array}{l}\text { Wambsganss et al. } \\
\text { (1993) [40] }\end{array}$ & $\begin{array}{l}\text { R113 in copper } \\
\text { circular } \\
\mathrm{D}=2.92\end{array}$ & $\begin{array}{l}\mathrm{G}=50-300 \\
\mathrm{q}^{\prime \prime}=0.9-91 \\
\mathrm{x}=0-0.9\end{array}$ & $\begin{array}{l}\text { Measurements on boiling heat transfer: } \\
\text { Nucleate boiling dominates the heat transfer } \\
\text { h is strongly dependent on q", and weakly dependent on } \mathrm{G} \text { and } \mathrm{x}\end{array}$ \\
\hline $\begin{array}{l}\text { Peng et al. (1995) } \\
\text { [41] }\end{array}$ & $\begin{array}{l}\text { methanol in stainless steel } \\
\text { rectangular } \\
\mathrm{D}_{\mathrm{h}}=0.255-0.646\end{array}$ & $\begin{array}{l}\mathrm{T}_{\mathrm{in}}=14-19 \\
\mathrm{v}=0.2-1.5\end{array}$ & $\begin{array}{l}\text { Experiments on boiling: } \\
\text { Liquid velocity and subcooling do not affect fully developed nucleate boiling } \\
\text { Greater subcooling increases velocity and suppresses initiation of flow boiling }\end{array}$ \\
\hline Cuta et al. (1996) [42] & $\begin{array}{l}\mathrm{R} 124 \text { in copper } \\
\text { rectangular }(\mathrm{N}=54) \\
\mathrm{D}_{\mathrm{h}}=0.425\end{array}$ & $\begin{array}{l}\operatorname{Re}=100-570 \\
q^{\prime \prime}=0-40 \\
x=0.04-0.6\end{array}$ & $\begin{array}{l}\text { Experiments on microchannel heat exchanger: } \\
\text { Nusselt number shows an increase with Re in single-phase flow, but is approximately } \\
\text { constant in two-phase flow }\end{array}$ \\
\hline $\begin{array}{l}\text { Mertz et al. (1996) } \\
\text { [43] }\end{array}$ & $\begin{array}{l}\text { water and } \mathrm{R} 141 \mathrm{~b} \text { in copper } \\
\text { rectangular } \\
\mathrm{W}=1,2,3 \mathrm{~mm}, \mathrm{H} / \mathrm{W}<3\end{array}$ & $\begin{array}{l}\mathrm{G}=50-300 \\
\mathrm{q}^{\prime \prime}=0-200 \\
\mathrm{P}=100-200\end{array}$ & $\begin{array}{l}\text { Experiments on flow boiling in narrow channels of planar heat exchanger elements: } \\
\text { Boiling curves and variations of heat transfer coefficient with local and average heat } \\
\text { fluxes are presented }\end{array}$ \\
\hline $\begin{array}{l}\text { Peng et al. (1996) } \\
\text { [44] }\end{array}$ & $\begin{array}{l}\text { methanol-water mixture in } \\
\text { stainless steel } \\
\text { rectangular } \\
\mathrm{D}_{\mathrm{h}}=0.133-0.343\end{array}$ & $\begin{array}{l}\mathrm{T}_{\mathrm{in}}=18-28 \\
\mathrm{v}=0.1-4.0\end{array}$ & $\begin{array}{l}\text { Experiments on flow boiling in binary mixtures: } \\
\text { h at onset of flow boiling and in partial nucleate boiling is greatly influenced by } \\
\text { concentration, microchannel dimensions, flow velocity and subcooling } \\
\text { These parameters have no significant effect on h in the fully nucleate boiling regime } \\
\text { - Mixtures with small concentrations of methanol augmented flow boiling heat transfer }\end{array}$ \\
\hline $\begin{array}{l}\text { Tran et al. (1996) } \\
\text { [27] }\end{array}$ & $\begin{array}{l}\mathrm{R} 12 \text { and } \mathrm{R} 113 \text { in brass } \\
\text { circular and rectangular } \\
\mathrm{D}_{\mathrm{h}}=2.4-2.46\end{array}$ & $\begin{array}{l}\mathrm{G}=44-832 \\
\mathrm{q}^{\prime \prime}=0.36-12.9 \\
\mathrm{P}=510-820 \\
\mathrm{x}=0.2-0.94\end{array}$ & $\begin{array}{l}\text { Boiling heat transfer measurements on circular and rectangular channels } \\
\text { Nucleate boiling dominates the heat transfer } \\
\text { h is independent of } \mathrm{x} \text { for } \mathrm{x}>0.2 \text {, and independent of } \mathrm{G}\end{array}$ \\
\hline
\end{tabular}




\begin{tabular}{|c|c|c|c|}
\hline Reference & $\begin{array}{c}\text { Fluid/Substrate, } \\
\text { Geometry, } D_{h}[\mathrm{~mm}], \mathbf{N}\end{array}$ & 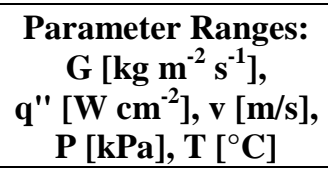 & Remarks \\
\hline $\begin{array}{l}\text { Kew and Cornwell } \\
\text { (1997) [45] }\end{array}$ & $\begin{array}{l}\mathrm{R} 141 \mathrm{~b} \\
\text { circular } \\
\mathrm{D}=1.39-3.69\end{array}$ & $\begin{array}{l}\mathrm{G}=150-1500 \\
\mathrm{q}^{\prime \prime}=1.0-10.0 \\
\mathrm{P}=100 \\
\mathrm{x}=-0.051 \text { to } 0.95\end{array}$ & $\begin{array}{l}\text { Measurements of heat transfer in different-sized channels: } \\
\text { Existing correlations do not perform well above a confinement number of } \\
\text { approximately } 0.5 \\
\text { Nucleate boiling correlations perform better }\end{array}$ \\
\hline $\begin{array}{l}\text { Peng and Wang } \\
\text { (1998) [46] }\end{array}$ & $\begin{array}{l}\text { methanol and water in } \\
\text { stainless steel } \\
\text { rectangular }(\mathrm{N}=1-6) \\
\mathrm{D}_{\mathrm{h}}=0.133-0.343\end{array}$ & $\begin{array}{l}\mathrm{Re}=50-3000 \\
\mathrm{q}^{\prime \prime}=0-50\end{array}$ & $\begin{array}{l}\text { Experiments in several microchannel geometries: } \\
\text { Microchannel size, shape, and configuration have significant effect on bubble } \\
\text { formation and heat transfer } \\
\text { Nucleate boiling is almost impossible in microchannels }\end{array}$ \\
\hline $\begin{array}{l}\text { Peng et al. (1998) } \\
\text { [47] }\end{array}$ & $\begin{array}{l}\text { water and methanol in } \\
\text { stainless steel } \\
\text { V-shaped }(\mathrm{N}=6) \\
\text { groove angles } 30-60^{\circ} \\
\mathrm{D}_{\mathrm{h}}=0.2-0.6\end{array}$ & $\begin{array}{l}\mathrm{v}=0.12-2.14 \\
\mathrm{q}^{\prime \prime}=0-100\end{array}$ & $\begin{array}{l}\text { Experiments on flow boiling: } \\
\text { Heat transfer and pressure drop are affected by flow velocity, subcooling, } \mathrm{D}_{\mathrm{h}} \text { and } \\
\text { groove angle } \\
\text { No bubbles are observed in microchannels during flow boiling, unlike in } \\
\text { conventional channels } \\
\text { Experiments indicate an optimum for } \mathrm{D}_{\mathrm{h}} \text { and groove angle }\end{array}$ \\
\hline $\begin{array}{l}\text { Peng et al. (1998) } \\
\text { [48] }\end{array}$ & rectangular microchannels & & $\begin{array}{l}\text { Modeling of nucleate boiling in microchannels: } \\
\text { Model considers bubble growth mechanism and microchannel geometry } \\
\text { A new dimensionless parameter is introduced to describe boiling conditions for } \\
\text { liquids }\end{array}$ \\
\hline $\begin{array}{l}\text { Ravigururajan (1998) } \\
\text { [49] }\end{array}$ & $\begin{array}{l}\text { R124 in copper } \\
\text { rectangular and diamond }(\mathrm{N} \\
=54) \\
\mathrm{D}_{\mathrm{h}}=0.425\end{array}$ & $\begin{array}{l}q^{\prime \prime}=0-70 \\
x=0-0.65\end{array}$ & $\begin{array}{l}\text { Experiments with two microchannel patterns (parallel and diamond): } \\
\text { h and pressure drop are functions of x, G, q", and surface superheat } \\
\text { h decreases by } 20-30 \% \text { for an increase in exit vapor quality from } 0.01 \text { to } 0.65\end{array}$ \\
\hline $\begin{array}{l}\text { Yan and Lin (1998) } \\
\text { [28] }\end{array}$ & $\begin{array}{l}\mathrm{R} 134 \mathrm{a} \text { in copper } \\
\text { circular }(\mathrm{N}=28) \\
\mathrm{D}=2.0\end{array}$ & $\begin{array}{l}\mathrm{G}=50-200 \\
\mathrm{q}^{\prime \prime}=0.5-2.0 \\
\mathrm{~T}_{\mathrm{sat}}=5-31 \\
\mathrm{x}=0.05-0.85\end{array}$ & $\begin{array}{l}\text { Experimental boiling heat transfer and pressure drop: } \\
\text { G Variation of } h \text { with } x \text { is not consistent, and depends on q", saturation temperature and } \\
\end{array}$ \\
\hline Bao et al. (2000) [32] & $\begin{array}{l}\text { R11, R123 in copper } \\
\text { circular } \\
\mathrm{D}=1.95\end{array}$ & $\begin{array}{l}\mathrm{G}=50-1800 \\
\mathrm{q}^{\prime \prime}=0.5-20 \\
\mathrm{P}=200-500 \\
\mathrm{x}=0.0-0.9\end{array}$ & $\begin{array}{l}\text { Experimental flow boiling heat transfer: } \\
\text { Nucleate boiling dominates the heat transfer } \\
\text { h is independent of } \mathrm{G} \text { and } \mathrm{x} \text {, and increases with q" and } \mathrm{P} \text { for the saturated regime }\end{array}$ \\
\hline $\begin{array}{l}\text { Zhao et al. (2000) } \\
{[50]}\end{array}$ & $\mathrm{CO}_{2}$ in stainless steel & $\begin{array}{l}\mathrm{G}=250-700 \\
\mathrm{q}^{\prime \prime}=0.8-2.5 \\
\mathrm{~T}_{\text {sat }}=5-15 \\
\mathrm{x}=0.05-0.3\end{array}$ & $\begin{array}{l}\text { Flow boiling measurements: } \\
\text { h is almost independent of q" and G } \\
\text { Heat transfer mechanism depends on size and geometry of channels }\end{array}$ \\
\hline
\end{tabular}




\begin{tabular}{|c|c|c|c|}
\hline Reference & $\begin{array}{l}\text { Fluid/Substrate, } \\
\text { Geometry, } D_{h}[\mathrm{~mm}], \mathbf{N}\end{array}$ & 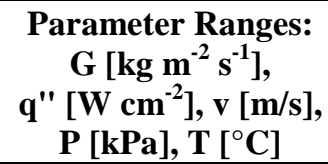 & Remarks \\
\hline $\begin{array}{l}\text { Lee and Lee (2001) } \\
{[25]}\end{array}$ & $\begin{array}{l}\text { R113 in stainless steel } \\
\text { R12 and R134a in brass } \\
\text { rectangular and circular } \\
\mathrm{D}_{\mathrm{h}}=2.40-2.92\end{array}$ & $\begin{array}{l}\mathrm{G}=50-200 \\
\mathrm{q}^{\prime \prime}=0.0-1.5 \\
\mathrm{Re}=100-1000 \\
x=0.15-0.75\end{array}$ & $\begin{array}{l}\text { Flow boiling experiment and correlation development: } \\
\text { Heat transfer dominated by convection } \\
\text { h is independent of q", and increases with } \mathrm{G} \text { and } \mathrm{x} \\
\text { Film flow heat transfer correlation applicable for low flow rate and Kandlikar } \\
\text { correlation applicable for high flow rate }\end{array}$ \\
\hline Lin et al. (2001) [29] & $\begin{array}{l}\text { water and R141b } \\
\text { circular } \\
\mathrm{D}=1\end{array}$ & $\begin{array}{l}\mathrm{Re}=300-2000 \\
\mathrm{q}^{\prime \prime}=1-115 \\
\mathrm{x}=0-1 \\
\mathrm{~T}_{\mathrm{sat}}=39-56\end{array}$ & $\begin{array}{l}\text { Heat transfer measurement in a microchannel: } \\
\text { h shows inconsistent behavior with respect to } \mathrm{x} \\
\text { h peaks between } 0.1 \text { and } 0.6 \text { vapor quality }\end{array}$ \\
\hline $\begin{array}{l}\text { Jacobi and Thome } \\
(2002)[51]\end{array}$ & $\mathrm{D}_{\mathrm{h}}=1.95$ & $\begin{array}{l}\mathrm{G}=150-450 \\
\mathrm{q}^{\prime \prime}=4-20 \\
\mathrm{P}=460 \\
\operatorname{Re}=1000-2500 \\
\mathrm{x}=0.0-0.8\end{array}$ & $\begin{array}{l}\text { Heat transfer model for microchannel flow: } \\
\text { The model considers thin film evaporation into elongated bubbles } \\
\text { - A time average of the different flow patterns is used to determine } h\end{array}$ \\
\hline $\begin{array}{l}\text { Warrier et al. (2002) } \\
{[52]}\end{array}$ & $\begin{array}{l}\text { FC-84 in aluminum } \\
\text { rectangular }(\mathrm{N}=5) \\
\mathrm{D}_{\mathrm{h}}=0.75\end{array}$ & $\begin{array}{l}\mathrm{G}=557-1600 \\
\mathrm{q}^{\prime \prime}=0.0-4.5 \\
\mathrm{x}=0.0-0.5\end{array}$ & $\begin{array}{l}\text { Experimental heat transfer and pressure drop measurements: } \\
\text { No significant maldistribution is found for 5-channel configuration } \\
\text { Flow pattern develops quickly into annular flow }\end{array}$ \\
\hline Yu et al. (2002) [53] & $\begin{array}{l}\text { Water in stainless steel } \\
\text { circular } \\
\mathrm{D}=2.98\end{array}$ & $\begin{array}{l}\mathrm{G}=50-200 \\
\mathrm{q}^{\prime \prime}=0-30 \\
\mathrm{~T}_{\text {in }}=20-80\end{array}$ & $\begin{array}{l}\text { Two-phase heat transfer, pressure drop and CHF } \\
\text { Nucleate boiling dominates over large G and x ranges }\end{array}$ \\
\hline $\begin{array}{l}\text { Zhang et al. (2002) } \\
\text { [54] }\end{array}$ & $\begin{array}{l}\text { water in silicon } \\
\text { rectangular }(\mathrm{N}=1-40) \\
\mathrm{D}_{\mathrm{h}}=0.025-0.060\end{array}$ & & $\begin{array}{l}\text { Measurement and modeling of two-phase flow in microchannels: } \\
\text { Boiling occurs in plasma etched silicon without excessive superheating }\end{array}$ \\
\hline $\begin{array}{l}\text { Agostini et al. (2003) } \\
\text { [31] }\end{array}$ & $\begin{array}{l}\mathrm{R}-134 \mathrm{a} \text { in aluminum } \\
\text { rectangular }(\mathrm{N}=11-18) \\
\mathrm{D}_{\mathrm{h}}=0.77-2.01\end{array}$ & $\begin{array}{l}\mathrm{G}=90-295 \\
\mathrm{q}^{\prime \prime}=0.6-3.2 \\
\mathrm{p}=405 \\
\mathrm{x}=0.26-1.0\end{array}$ & $\begin{array}{l}\text { Heat transfer measurements on mulitport small channel heat exchangers: } \\
\text { Reduced hydraulic diameter increases } \mathrm{h} \\
\text { - Maximum heat transfer coefficient at x between } 0.2-0.4\end{array}$ \\
\hline $\begin{array}{l}\text { Faulkner et al. (2003) } \\
\text { [55] }\end{array}$ & $\begin{array}{l}\text { water and nanofluid } \\
\text { rectangular }(\mathrm{N}=10) \\
\mathrm{D}_{\mathrm{h}}=0.93-1.71\end{array}$ & $\begin{array}{l}\mathrm{G}=201-278 \\
\operatorname{Re}=200-1000 \\
q^{\prime \prime}=0-275\end{array}$ & $\begin{array}{l}\text { Experiments in a heat exchanger with parallel channels: } \\
\text { Nanofluid is intended to reduce flow misdistribution between parallel channels } \\
\text { Nanofluid improves overall heat transfer rate }\end{array}$ \\
\hline $\begin{array}{l}\text { Haynes and Fletcher } \\
\text { (2003) [134] }\end{array}$ & $\begin{array}{l}\text { R11 and R123 in copper } \\
\text { circular } \\
\mathrm{D}_{\mathrm{h}}=0.92-1.95\end{array}$ & $\begin{array}{l}\mathrm{G}=110-1840 \\
\mathrm{q}^{\prime \prime}=1.1-17 \\
\mathrm{x}=-0.2-1.0\end{array}$ & $\begin{array}{l}\text { Subcooled and saturated flow boiling measurements: } \\
\text { Nucleate boiling heat transfer outweighs convection after ONB } \\
\text { No evidence for suppression of the nucleate boiling due to convection is found }\end{array}$ \\
\hline
\end{tabular}




\begin{tabular}{|c|c|c|c|}
\hline Reference & $\begin{array}{c}\text { Fluid/Substrate, } \\
\text { Geometry, } D_{h}[\mathrm{~mm}], \mathbf{N}\end{array}$ & 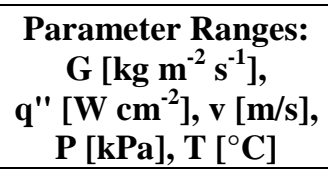 & Remarks \\
\hline $\begin{array}{l}\text { Qu and Mudawar } \\
(2003)[56]\end{array}$ & $\begin{array}{l}\text { water in copper } \\
\text { rectangular }(\mathrm{N}=21) \\
\mathrm{D}_{\mathrm{h}}=0.349\end{array}$ & $\begin{array}{l}\mathrm{G}=135-402 \\
\mathrm{q}^{\prime \prime}=0-150 \\
\mathrm{x}=0.0-0.15\end{array}$ & $\begin{array}{l}\text { Experimental two-phase heat transfer: } \\
\text { h decreases with increasing } \mathrm{x} \\
11 \text { previous correlations do not predict heat transfer adequately }\end{array}$ \\
\hline $\begin{array}{l}\text { Qu and Mudawar } \\
\text { (2003) [57] }\end{array}$ & $\begin{array}{l}\text { water in copper } \\
\text { rectangular }(\mathrm{N}=21) \\
\mathrm{D}_{\mathrm{h}}=0.349\end{array}$ & $\begin{array}{l}\mathrm{G}=135-402 \\
\mathrm{q}^{\prime \prime}=0-150 \\
\mathrm{x}=0.0-0.15\end{array}$ & $\begin{array}{l}\text { Annular flow model to predict saturated flow boiling heat transfer: } \\
\text { Laminar liquid and vapor flow, droplet entrainment, and deposition effects are } \\
\text { included in the model } \\
\text { Mean average error between correlation and measured data is } 13.3 \%\end{array}$ \\
\hline $\begin{array}{l}\text { Sumith et al. (2003) } \\
\text { [58] }\end{array}$ & $\begin{array}{l}\text { water in stainless steel } \\
\text { circular } \\
D_{h}=1.45\end{array}$ & $\begin{array}{l}\mathrm{G}=23.4-152.7 \\
\mathrm{q}^{\prime \prime}=1-71.5 \\
\mathrm{x}=0-0.8\end{array}$ & $\begin{array}{l}\text { Measurement of flow boiling heat transfer in vertical tube: } \\
\text { Existing correlations largely under-predict the heat transfer } \\
\text { Katto's CHF correlation works well }\end{array}$ \\
\hline $\begin{array}{l}\text { Balsubramanian and } \\
\text { Kandlikar (2004) } \\
{[135]}\end{array}$ & $\begin{array}{l}\mathrm{R} 113, \mathrm{R} 141 \mathrm{~b}, \mathrm{R} 123 \\
\text { circular } \\
\mathrm{D}_{\mathrm{h}}=0.19-2.92\end{array}$ & $\begin{array}{l}\mathrm{G}=50-570 \\
\mathrm{q}=0.55-9.1 \\
\operatorname{Re}=72-2955 \\
x=0-1\end{array}$ & $\begin{array}{l}\text { Extension of flow boiling correlation to transition and laminar flow: } \\
\text { Kandlikar (1991) correlation is modified for new measurements } \\
\text { Reynolds number of transition in microchannels is not well defined }\end{array}$ \\
\hline $\begin{array}{l}\text { Campbell and } \\
\text { Kandlikar (2004) [59] }\end{array}$ & $\begin{array}{l}\text { water in copper } \\
\text { circular } \\
\mathrm{D}_{\mathrm{h}}=0.706\end{array}$ & $\begin{array}{l}\mathrm{G}=44-3070 \\
\mathrm{q}^{\prime \prime}=20-30 \\
\mathrm{x}_{\text {out }}=0.8-1.0\end{array}$ & $\begin{array}{l}\text { Experiments on heat transfer and pressure drop in flow boiling: } \\
\text { h increases with wall superheat until dryout occurs } \\
\text { Pressure drop in channels is nearly constant for dryout conditions } \\
\text { Dominant frequency of the pressure drop decreases with increasing surface } \\
\text { temperature }\end{array}$ \\
\hline $\begin{array}{l}\text { Henning et al. (2004) } \\
\text { [60] }\end{array}$ & $\begin{array}{l}\text { water in silicon } \\
\text { straight and wavy fins }(\mathrm{N}= \\
462-618)\end{array}$ & $\begin{array}{l}\mathrm{m}=0-64 \mathrm{~kg} / \mathrm{h} \\
\mathrm{Q}_{\mathrm{el}}=0-3 \mathrm{~kW}\end{array}$ & $\begin{array}{l}\text { Microchannel heat exchanger flow boiling measurements for straight and sinusoidal } \\
\text { fins: } \\
\text { - Sinusoidal path increases } \mathrm{h} \\
\text { - Short microchannels outperform longer microchannels }\end{array}$ \\
\hline $\begin{array}{l}\text { Hetsroni et al. (2004) } \\
\text { [5] }\end{array}$ & $\begin{array}{l}\text { water in silicon } \\
\text { triangular }(\mathrm{N}=17-26) \\
\mathrm{D}_{\mathrm{h}}=0.103-0.161\end{array}$ & $\begin{array}{l}\mathrm{Re}=8-42 \\
\mathrm{q}^{\prime \prime}=5.1-50\end{array}$ & $\begin{array}{l}\text { Experiments on convective flow boiling in parallel channels: } \\
\text { Different flow patterns are observed simultaneously in various microchannels } \\
\text { Flow instabilities are observed } \\
\text { Surface temperature is almost constant over the entire surface during boiling }\end{array}$ \\
\hline $\begin{array}{l}\text { Qu and Mudawar } \\
(2004)[61]\end{array}$ & $\begin{array}{l}\text { water in copper } \\
\text { rectangular }(\mathrm{N}=21) \\
\mathrm{D}_{\mathrm{h}}=0.35\end{array}$ & $\begin{array}{l}\mathrm{G}=135-402 \\
\mathrm{q}^{\prime \prime}=0-250 \\
\mathrm{x}=0-0.2\end{array}$ & $\begin{array}{l}\text { Experiments on two-phase flow in microchannels: } \\
\text { Lockhardt Martinelli correlation gives acceptable predictions for pressure drop } \\
\text { measurements } \\
\text { Investigated heat transfer correlations are deemed unsuitable to predict } \\
\text { entrainment in the annular regime are observed }\end{array}$ \\
\hline
\end{tabular}




\begin{tabular}{|c|c|c|c|}
\hline Reference & $\begin{array}{l}\text { Fluid/Substrate, } \\
\text { Geometry, } \mathrm{D}_{\mathrm{h}}[\mathrm{mm}], \mathbf{N}\end{array}$ & $\begin{array}{l}\text { Parameter Ranges: } \\
\text { G }\left[\mathrm{kg} \mathrm{m}^{-2} \mathrm{~s}^{-1}\right] \\
\mathbf{q}^{\prime \prime}\left[\mathrm{W} \mathrm{cm} \mathbf{~ c m}^{-2}\right], \mathbf{v}[\mathbf{m} / \mathbf{s}] \\
\mathbf{P}[\mathbf{k P a}], \mathbf{T}\left[{ }^{\circ} \mathbf{C}\right]\end{array}$ & Remarks \\
\hline $\begin{array}{l}\text { Steinke and Kandlikar } \\
\text { (2004) [35] }\end{array}$ & $\begin{array}{l}\text { r water in copper } \\
\text { rectangular }(\mathrm{N}=6) \\
\mathrm{D}_{\mathrm{h}}=0.207\end{array}$ & $\begin{array}{l}\mathrm{G}=157-1782 \\
\operatorname{Re}=116-1318 \\
\mathrm{q}=0-93 \\
\mathrm{x}=0-1\end{array}$ & $\begin{array}{l}\text { Experimental flow boiling in parallel microchannels: } \\
\text { Single-phase measurements agree with conventional theory } \\
\text { Flow reversal exists in parallel microchannels } \\
\text { h decreases with increasing x } \\
\text { Nucleate boiling dominates the heat transfer }\end{array}$ \\
\hline $\begin{array}{l}\text { Thome et al. (2004) } \\
\text { [62] }\end{array}$ & microchannels & & $\begin{array}{l}\text { Elongated bubble model proposed: } \\
\text { Model describes transient variation of the local } \mathrm{h} \\
\text { - Fluid flow can be divided into liquid slug, elongated bubble and vapor slug } \\
\text { h is obtained by time averaging }\end{array}$ \\
\hline $\begin{array}{l}\text { Thome et al. (2004) } \\
\text { [63] }\end{array}$ & $\begin{array}{l}\mathrm{R} 11, \mathrm{R} 12, \mathrm{R} 113, \mathrm{R} 123 \\
\mathrm{R} 134 \mathrm{a}, \mathrm{R} 141 \mathrm{~b}, \mathrm{CO}_{2} \\
\mathrm{D}_{\mathrm{h}}=0.77-3.1\end{array}$ & $\begin{array}{l}\mathrm{G}=50-564 \\
\mathrm{q}^{\prime \prime}=0.5-17.8 \\
\mathrm{P}=124-5777 \\
\mathrm{x}=0.01-0.99\end{array}$ & $\begin{array}{l}\text { Comparison of elongated bubble model with experiments } \\
67 \% \text { of the data are predicted within } \pm 30 \%\end{array}$ \\
\hline $\begin{array}{l}\text { Zhang et al. (2004) } \\
\text { [64] }\end{array}$ & $\begin{array}{l}\text { water, } \mathrm{R} 11, \mathrm{R} 12, \mathrm{R} 113 \\
\text { circular and rectangular } \\
\mathrm{D}_{\mathrm{h}}=0.78-6.0\end{array}$ & $\begin{array}{l}\mathrm{G}=23.4-2939 \\
\mathrm{q}^{\prime \prime}=2.98-251.1 \\
\mathrm{p}=101-866\end{array}$ & $\begin{array}{l}\text { Model for flow boiling: } \\
\text { Modification of the Chen correlation } \\
\text { Correlation is applicable for } \mathrm{x}<0.7\end{array}$ \\
\hline $\begin{array}{l}\text { Lee and Mudawar } \\
(2005)[65]\end{array}$ & $\begin{array}{l}\mathrm{R} 134 \mathrm{a} \text { in copper } \\
\text { rectangular }(\mathrm{N}=53) \\
\mathrm{D}_{\mathrm{h}}=0.35\end{array}$ & $\begin{array}{l}\mathrm{G}=127-654 \\
\mathrm{q}^{\prime \prime}=32-94 \\
\mathrm{~T}_{\mathrm{sat}}=-18-25 \\
\mathrm{x}=0.0-1.0\end{array}$ & $\begin{array}{l}\text { Experiments on refrigerant two-phase flow heat transfer: } \\
\text { - Nucleate boiling occurs only at low x } \\
\text { - Annular flow regime is dominant for medium and high } \mathrm{x} \\
\text { - Model proposed to predict } \mathrm{h} \text { is introduced }\end{array}$ \\
\hline $\begin{array}{l}\text { Lie and Lin (2005) } \\
{[66]}\end{array}$ & $\begin{array}{l}\mathrm{R} 134 \text { in copper } \\
\text { annular } \\
\mathrm{D}_{\mathrm{h}}=2.0-4.0\end{array}$ & $\begin{array}{l}\mathrm{q}^{\prime \prime}=0-5 \\
\mathrm{~T}_{\text {sat }}=10-15\end{array}$ & $\begin{array}{l}\text { Flow measurement and visualization in a narrow annular duct: } \\
\text { h increases with increase in q" } \\
\text { G and saturation temperature effects are far smaller } \\
\text { Correlations proposed to predict bubble diameter, frequency and heat transfer }\end{array}$ \\
\hline Liu et al. (2005) [67] & $\begin{array}{l}\text { water in copper } \\
\text { rectangular }(\mathrm{N}=25) \\
\mathrm{D}_{\mathrm{h}}=0.384\end{array}$ & $\begin{array}{l}\mathrm{G}=31-90 \\
\operatorname{Re}=288-1050 \\
\mathrm{q}^{\prime \prime}=10-70\end{array}$ & $\begin{array}{l}\text { Measurement of ONB in microchannels and development of analytical model: } \\
\text { ONB depends on inlet conditions, contact angle, velocity, subcooling, pressure and } \\
\text { microchannel dimensions }\end{array}$ \\
\hline $\begin{array}{l}\text { Saitoh et al. (2005) } \\
\text { [24] }\end{array}$ & $\begin{array}{l}\text { R134a } \\
\text { circular } \\
\mathrm{D}_{\mathrm{h}}=0.51,1.12,3.1\end{array}$ & $\begin{array}{l}\mathrm{G}=150-450 \\
\mathrm{q}^{\prime \prime}=0.5-3.9 \\
\mathrm{~T}_{\mathrm{sat}}=5-15 \\
\mathrm{x}=0.0-0.2\end{array}$ & $\begin{array}{l}\text { Measurements of the effect of tube diameter on boiling heat transfer: } \\
\text { As tube diameter decreases, the flow inside the tube approaches homogeneous flow } \\
\text { Effect of forced convective heat transfer decreases with decreasing tube diameter }\end{array}$ \\
\hline $\begin{array}{l}\text { Zhang et al. (2005) } \\
\text { [68] }\end{array}$ & $\begin{array}{l}\text { water, } \mathrm{R} 11, \mathrm{R} 12, \mathrm{R} 113 \\
\text { circular and rectangular } \\
\mathrm{D}_{\mathrm{h}}=0.78-6.0\end{array}$ & $\begin{array}{l}\mathrm{G}=23.4-560 \\
q^{\prime \prime}=0.4-80.3 \\
\mathrm{p}=101-560\end{array}$ & $\begin{array}{l}\text { Modeling paper for flow boiling in small channels for low Reynolds numbers: } \\
\text { Uses superposition of nucleate boiling and forced convection } \\
\text { Mean deviation and RMS error are } 19.1 \% \text { and } 24.3 \% \text {, respectively }\end{array}$ \\
\hline
\end{tabular}




\begin{tabular}{|c|c|c|c|}
\hline Reference & $\begin{array}{c}\text { Fluid/Substrate, } \\
\text { Geometry, } D_{\mathrm{h}}[\mathrm{mm}], \mathbf{N}\end{array}$ & 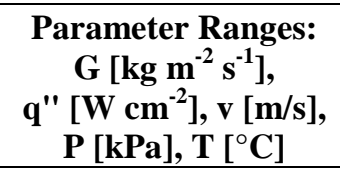 & Remarks \\
\hline $\begin{array}{l}\text { Chen and Garimella } \\
\text { (2006) [69] }\end{array}$ & $\begin{array}{l}\text { FC-77 in silicon } \\
\text { rectangular }(\mathrm{N}=24) \\
\mathrm{D}_{\mathrm{h}}=0.39\end{array}$ & $\begin{array}{l}\mathrm{G}=250-440 \\
\mathrm{q}^{\prime \prime}=2-80 \\
\mathrm{~T}_{\mathrm{sat}}=97 \\
\mathrm{x}=0.01-0.99\end{array}$ & $\begin{array}{l}\text { Experiments on heat transfer, pressure drop and flow visualization: } \\
\text { At high q" partial dryout occurs leading to temperature fluctuations on the wall } \\
\text { h best predicted by the Cooper (1984) correlation (pool boiling) } \\
\text { Flow instabilities do not affect the averaged pressure drop }\end{array}$ \\
\hline $\begin{array}{l}\text { Chen and Garimella } \\
\text { (2006) [70] }\end{array}$ & $\begin{array}{l}\text { FC-77 in silicon } \\
\text { Rectangular }(\mathrm{N}=10) \\
\mathrm{D}_{\mathrm{h}}=0.839\end{array}$ & $\begin{array}{l}\mathrm{q}^{\prime \prime}=0-14 \\
\mathrm{~T}_{\text {sat }}=70-97\end{array}$ & $\begin{array}{l}\text { Effect of dissolved air on subcooled flow boiling } \\
\text { Boiling incipience temperature was reduced drastically if dissolved gas was present } \\
\text { Early boiling incipience for the undegassed liquid resulted in higher h at low q" }\end{array}$ \\
\hline Yen et al. (2006) [71] & $\begin{array}{l}\text { R123 in glass } \\
\text { circular and rectangular } \\
\mathrm{D}_{\mathrm{h}}=0.210-0.214\end{array}$ & $\begin{array}{l}\mathrm{G}=100-800 \\
\mathrm{q}^{\prime \prime}=0-5 \\
\mathrm{x}=0.0-0.8\end{array}$ & $\begin{array}{l}\text { Boiling heat transfer measurements with different cross-sections: } \\
\mathrm{h} \text { in rectangular tubes is higher than in circular ones due to the corners acting as } \\
\text { nucleation sites } \\
\text { h increases with decreasing } \mathrm{x}\end{array}$ \\
\hline Yun et al. (2006) [33] & $\begin{array}{l}\mathrm{R} 410 \mathrm{~A} \text { in aluminum } \\
\text { rectangular } \\
\mathrm{D}_{\mathrm{h}}=1.36-1.44\end{array}$ & $\begin{array}{l}\mathrm{G}=200-400 \\
\mathrm{q}^{\prime \prime}=1-2 \\
\mathrm{~T}_{\text {sat }}=0,5,10 \\
\mathrm{x}=0-0.85\end{array}$ & $\begin{array}{l}\text { Evaporative heat transfer and pressure drop measurements: } \\
\text { Heat transfer results diverge from conventional-sized channels } \\
\text { Saturation temperature has small impact on } \mathrm{h} \\
\text { h peaks at a } \mathrm{x} \approx 0.5\end{array}$ \\
\hline $\begin{array}{l}\text { Calame et al. (2007) } \\
\text { [72] }\end{array}$ & $\begin{array}{l}\text { water in silicon } \\
\text { rectangular }(\mathrm{N}=7) \\
\mathrm{D}_{\mathrm{h}}=0.737\end{array}$ & $\begin{array}{l}\mathrm{m}=0.72-3.81 / \mathrm{min} \\
\mathrm{q}^{\prime \prime}=0-5000 \text { (with } \\
\text { respect to base area) }\end{array}$ & $\begin{array}{l}\text { Microchannels used to cool extremely high heat flux devices: } \\
\text { Silicon seen to show comparable thermal performance to copper as substrate } \\
\text { Thermal expansion coefficient of chip and heat sink are matched }\end{array}$ \\
\hline $\begin{array}{l}\text { Chen and Garimella } \\
\text { (2007) [73] }\end{array}$ & $\begin{array}{l}\text { FC-77 in silicon } \\
\text { rectangular }(\mathrm{N}=10) \\
\mathrm{D}_{\mathrm{h}}=0.839\end{array}$ & $\begin{array}{l}\mathrm{q}^{\prime \prime}=0.9-21 \\
\mathrm{~T}_{\mathrm{sat}}=97\end{array}$ & $\begin{array}{l}\text { Experimental flow boiling heat transfer: } \\
\text { CHF increases with flow rate } \\
\text { Dryout first occurs at the exit of the microchannels } \\
\text { Nucleate boiling correlation of Cooper (1984) was found to agree well with } \\
\text { measured h }\end{array}$ \\
\hline $\begin{array}{l}\text { Harirchian and } \\
\text { Garimella (2008) [74] }\end{array}$ & $\begin{array}{l}\text { FC-77 in silicon } \\
\text { rectangular }(\mathrm{N}=10-60) \\
\mathrm{D}_{\mathrm{h}}=0.16-0.57 \mathrm{~mm}\end{array}$ & $\begin{array}{l}G=250-1600 \\
x=0-1 \\
q^{\prime \prime}=0-30\end{array}$ & $\begin{array}{l}\text { Heat transfer and pressure drop measurements for different channel sizes: } \\
\text { Boiling curves are independent of } \mathrm{G} \text { in nucleate boiling regime } \\
\text { Pressure drop increases rapidly with q" in the two-phase region }\end{array}$ \\
\hline $\begin{array}{l}\text { Huh and } \operatorname{Kim}(2007) \\
{[75]}\end{array}$ & $\begin{array}{l}\text { water in Parylene C Dimer } \\
\text { rectangular } \\
\mathrm{D}_{\mathrm{h}}=0.1\end{array}$ & $\begin{array}{l}\mathrm{G}=90-267 \\
\mathrm{q}^{\prime \prime}=20-50 \\
\mathrm{P}_{\mathrm{sat}}=100 \\
\mathrm{x}=0.0-0.4\end{array}$ & $\begin{array}{l}\text { Boiling heat transfer, pressure drop, and flow patterns: } \\
\text { Standard correlations do not predict h reliably } \\
\text { h is nearly independent of G and } \mathrm{x}\end{array}$ \\
\hline $\begin{array}{l}\text { Kuznetsov and } \\
\text { Shamirzaev (2007) } \\
{[76]}\end{array}$ & $\begin{array}{l}\text { R21 in stainless steel } \\
\text { rectangular } \\
\mathrm{D}_{\mathrm{h}}=2.55\end{array}$ & $\begin{array}{l}\mathrm{G}=50-215 \\
\mathrm{q}^{\prime \prime}=0.6 \\
\mathrm{x}=0.05-0.97\end{array}$ & $\begin{array}{l}\text { Boiling heat transfer measurements: } \\
\text { Main heat transfer mode for large } G \text { is convective boiling } \\
\text { Modified Liu and Winterton [132] and Balasubramanian and Kandlikar [108] model } \\
\text { describes convective heat transfer well }\end{array}$ \\
\hline
\end{tabular}




\begin{tabular}{|c|c|c|c|}
\hline Reference & $\begin{array}{l}\text { Fluid/Substrate, } \\
\text { Geometry, } D_{h}[\mathrm{~mm}], \mathbf{N}\end{array}$ & 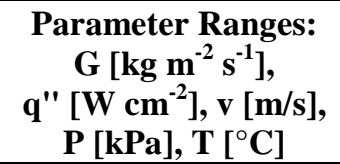 & Remarks \\
\hline $\begin{array}{l}\text { Liu and Garimella } \\
\text { (2007) [77] }\end{array}$ & $\begin{array}{l}\text { water in copper } \\
\text { rectangular }(\mathrm{N}=25) \\
\mathrm{D}_{\mathrm{h}}=0.384-0.796\end{array}$ & $\begin{array}{l}\mathrm{G}=221-1283 \\
\mathrm{q}^{\prime \prime}=0-129 \\
\mathrm{x}=0.0-0.2\end{array}$ & $\begin{array}{l}\text { Flow boiling experiments in parallel microchannels: } \\
\text { - Subcooled boiling can be predicted with conventional correlations } \\
\text { - New superposition model proposed for saturated boiling regime }\end{array}$ \\
\hline $\begin{array}{l}\text { Peters and Kandlikar } \\
\text { (2007) [78] }\end{array}$ & $\begin{array}{l}\text { water, } \mathrm{R} 123 \\
\text { circular, rectangular } \\
\mathrm{D}_{\mathrm{h}}=0.21-0.23\end{array}$ & $\begin{array}{l}\mathrm{G}=86-800 \\
\mathrm{q}^{\prime \prime}=2.5-192 \\
\mathrm{x}=0-0.7\end{array}$ & $\begin{array}{l}\text { Update of previous correlation: } \\
\text { Nucleate boiling term agrees better than convective boiling term } \\
\text { Experiments with surface modifications in the channels deviate from model }\end{array}$ \\
\hline Qi et al. (2007) [79] & $\begin{array}{l}\text { nitrogen in stainless steel } \\
\text { circular } \\
\mathrm{D}_{\mathrm{h}}=0.53-1.93\end{array}$ & $\begin{array}{l}\mathrm{G}=440-3000 \\
\mathrm{q}^{\prime \prime}=5.1-21.4 \\
\mathrm{P}=140-890\end{array}$ & $\begin{array}{l}\text { Measurements of flow boiling heat transfer and CHF: } \\
\text { At low G nucleate boiling dominates heat transfer } \\
\text { At high G convective boiling dominates heat transfer } \\
\text { CHF is higher than for conventional-sized channels }\end{array}$ \\
\hline $\begin{array}{l}\text { Ribatski et al. (2007) } \\
\text { [80] }\end{array}$ & $\begin{array}{l}\text { acetone in silicon } \\
\text { triangular }(\mathrm{N}=10) \\
\mathrm{D}_{\mathrm{h}}=0.155\end{array}$ & $\begin{array}{l}\mathrm{G}=60-500 \\
\mathrm{q}^{\prime \prime}=12-30.6\end{array}$ & $\begin{array}{l}\text { Application of the three zone model in [62] to the experimental data of Xu et al. [81]: } \\
\text { A new set of parameters for the three zone model is derived }\end{array}$ \\
\hline $\begin{array}{l}\text { Saitoh et al. (2007) } \\
\text { [82] }\end{array}$ & $\begin{array}{l}\mathrm{R}-134 \mathrm{a} \\
\text { circular } \\
\mathrm{D}_{\mathrm{h}}=0.5-11 \\
\end{array}$ & $\begin{array}{l}\mathrm{x}=0-1 \\
2224 \text { data points }\end{array}$ & $\begin{array}{l}\text { Development of a Chen-type [125] correlation for flow boiling heat transfer: } \\
\text { Effect of diameter is included in the Weber number } \\
\text { Annular model is developed to predict dryout }\end{array}$ \\
\hline $\begin{array}{l}\text { Schneider et al. } \\
\text { (2007) [83] }\end{array}$ & $\begin{array}{l}\mathrm{R} 123 \text { in silicon } \\
\text { rectangular }(\mathrm{N}=5) \\
\mathrm{D}_{\mathrm{h}}=0.227\end{array}$ & $\begin{array}{l}\mathrm{G}=622-1368 \\
\mathrm{q}^{\prime \prime}=0-21.3 \\
\mathrm{~T}_{\text {sat }}=38-80\end{array}$ & $\begin{array}{l}\text { Experiments on cavitation and boiling in refrigerant: } \\
\text { Cavitating flows significantly enhance } \mathrm{h} \text { (up to } 84 \% \text { ) } \\
\text { After boiling initiates, the deviation between cavitating and non-cavitating flows } \\
\text { diminishes with q" } \\
\text { No temperature overshoot observed for nucleate boiling } \\
\text { In cavitating flows convective boiling dominates compared to nucleate boiling in } \\
\text { non-cavitating flows at low } \mathrm{x}\end{array}$ \\
\hline $\begin{array}{l}\text { Wang et al. (2007) } \\
\text { [84] }\end{array}$ & & & $\begin{array}{l}\text { Investigation of an evaporating thin film in a microchannel: } \\
\text { Micro region (film is less than } 1 \mu \mathrm{m} \text { thick) accounts for } 50 \% \text { of the total heat transfer } \\
\text { Contribution of thin film heat transfer is inversely proportional to the channel size }\end{array}$ \\
\hline $\begin{array}{l}\text { Bertsch et al. (2008) } \\
{[30]}\end{array}$ & $\begin{array}{l}\mathrm{R} 134 \mathrm{a} \text { in copper } \\
\text { rectangular }(\mathrm{N}=17-33) \\
\mathrm{D}_{\mathrm{h}}=0.54-1.089\end{array}$ & $\begin{array}{l}\mathrm{G}=20.3-170 \\
\mathrm{q}^{\prime \prime}=0-20 \\
\mathrm{~T}_{\mathrm{sat}}=8-30 \\
\mathrm{x}=-0.2-0.95\end{array}$ & $\begin{array}{l}\text { Measurement of heat transfer in small channels: } \\
\text { h strongly depends on q" and } \mathrm{x} \\
\text { h peaks at a vapor quality of } \sim 0.2 \\
\text { Effect of saturation pressure is small }\end{array}$ \\
\hline $\begin{array}{l}\text { Lee and Garimella } \\
(2008) \text { [85] }\end{array}$ & $\begin{array}{l}\text { water in silicon } \\
\text { rectangular }(\mathrm{N}=10-60) \\
\mathrm{D}_{\mathrm{h}}=0.163-0.571\end{array}$ & $\mathrm{q}^{\prime \prime}=0-230$ & $\begin{array}{l}\text { Experiments on saturated flow boiling heat transfer and pressure drop: } \\
\text { Existing correlations in the literature do not match pressure drop and heat transfer } \\
\text { measurements } \\
\text { New correlations for pressure drop and heat transfer are proposed and show good } \\
\text { agreement with the measured data }\end{array}$ \\
\hline
\end{tabular}




\begin{tabular}{|c|c|c|c|}
\hline Reference & $\begin{array}{l}\text { Fluid/Substrate, } \\
\text { Geometry, } \mathrm{D}_{\mathrm{h}}[\mathrm{mm}], \mathbf{N}\end{array}$ & 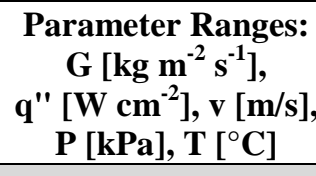 & Remarks \\
\hline \multicolumn{4}{|r|}{ Pressure Drop } \\
\hline $\begin{array}{l}\text { Bowers and Mudawa1 } \\
\text { (1994) [86] }\end{array}$ & $\begin{array}{l}\mathrm{R} 113 \text { in copper } \\
\text { circular }(\mathrm{N}=17) \\
\mathrm{D}=0.51,2.45\end{array}$ & $\begin{array}{l}\mathrm{q}^{\prime \prime}=0-200 \\
\Delta \mathrm{T}=10-32\end{array}$ & $\begin{array}{l}\text { Pressure drop model developed and predictions compared to experiments: } \\
\text { Major contributor to pressure drop identified as acceleration resulting from } \\
\text { evaporation } \\
\text { Compressibility effect important for microchannel when Mach number }>0.22 \\
\text { Channel erosion effects more predominant in microchannels than in minichannels }\end{array}$ \\
\hline $\begin{array}{l}\text { Triplett et al. (1999) } \\
\text { [87] }\end{array}$ & $\begin{array}{l}\text { Air-water mixture in glass } \\
\text { circular and triangular } \\
D_{h}=1.1-1.5\end{array}$ & $\mathrm{v}=0.02-80$ & $\begin{array}{l}\text { Frictional pressure drop measured and compared with various two-phase models: } \\
\text { Models and correlations over-predict channel void fraction and pressure drop in } \\
\text { annular flow pattern } \\
\text { Annular-flow interface momentum transfer and wall friction in microchannels } \\
\text { significantly different from those in larger channels }\end{array}$ \\
\hline \multicolumn{2}{|c|}{$\begin{array}{l}\text { Tran et al. (2000) [88] R113 in stainless steel } \\
\text { rectangular } \\
D_{h}=0.78-3.63\end{array}$} & $\begin{array}{l}\mathrm{G}=33-832 \\
\mathrm{q}^{\prime \prime}=0.2-12.9 \\
\mathrm{x}=0.02-0.95\end{array}$ & $\begin{array}{l}\text { Experimental two-phase pressure drop: } \\
\text { Conventional correlations failed to predict the pressure drop } \\
\text { New correlation developed considering surface tension and channel size }\end{array}$ \\
\hline $\begin{array}{l}\text { Lee and Mudawar } \\
(2005)[89]\end{array}$ & $\begin{array}{l}\text { R134a in copper } \\
\text { rectangular }(\mathrm{N}=53) \\
\mathrm{D}_{\mathrm{h}}=0.35\end{array}$ & $\begin{array}{l}\mathrm{G}=127-654 \\
\mathrm{q}^{\prime \prime}=32-94 \\
\mathrm{~T}_{\text {sat }}=-18-25 \\
\mathrm{x}=0.0-1.0\end{array}$ & $\begin{array}{l}\text { Experiments on refrigerant two-phase flow: } \\
\text { Pressure drop poorly predicted by homogeneous equilibrium and separated flow } \\
\text { models } \\
\text { New models incorporated liquid viscosity and surface tension } \\
\text { Throttling valve in refrigeration system suppresses large pressure oscillations }\end{array}$ \\
\hline $\begin{array}{l}\text { Lee and Mudawar } \\
\text { (2006) [90] }\end{array}$ & $\begin{array}{l}\mathrm{R} 134 \mathrm{a} \text { in copper } \\
\text { rectangular }(\mathrm{N}=53) \\
\mathrm{D}_{\mathrm{h}}=0.35\end{array}$ & $\begin{array}{l}\mathrm{G}=127-654 \\
\mathrm{q}^{\prime \prime}=32-94 \\
\mathrm{~T}_{\text {sat }}=-18-25 \\
\mathrm{x}=0.26-0.87\end{array}$ & $\begin{array}{l}\text { Experiments and optimization of microchannel evaporators: } \\
\text { h increases with G } \\
\text { Microscale refrigeration requires that the compressor inlet be free of liquid for } \\
\text { common compressors } \\
\text { Channels with high aspect ratio combine good heat transfer and low pressure drop }\end{array}$ \\
\hline $\begin{array}{l}\text { Hwang and Kim } \\
\text { (2006) [91] }\end{array}$ & $\begin{array}{l}\text { R134a in stainless steel } \\
\text { circular } \\
D_{h}=0.244-0.792\end{array}$ & $\begin{array}{l}\mathrm{G}=140-950 \\
\mathrm{Re}=200-3000 \\
\mathrm{x}=0.08-0.95\end{array}$ & $\begin{array}{l}\text { Flow boiling pressure drop in microtubes: } \\
\text { Pressure drop increases with } x \text { and } G \text { and decreases with diameter } \\
\text { Existing correlations failed to predict the pressure drop, so a new form of the } \\
\text { Lockhart-Martinelli correlation was introduced }\end{array}$ \\
\hline \multicolumn{4}{|r|}{ Critical Heat Flux } \\
\hline $\begin{array}{l}\text { Bowers and Mudawa1 } \\
\text { (1994) [92] }\end{array}$ & $\begin{array}{l}\text { R113 in copper } \\
\text { circular } \\
\mathrm{D}=0.51 \text { and } 2.45\end{array}$ & $\begin{array}{l}\mathrm{q}^{\prime \prime}=0-200 \\
\Delta \mathrm{T}=10-32\end{array}$ & $\begin{array}{l}\text { Experiments on boiling and two-phase flow: } \\
\text { CHF correlation for mini and microchannels developed }\end{array}$ \\
\hline
\end{tabular}




\begin{tabular}{|c|c|c|c|}
\hline Reference & $\begin{array}{l}\text { Fluid/Substrate, } \\
\text { Geometry, } D_{h}[\mathrm{~mm}], \mathbf{N}\end{array}$ & 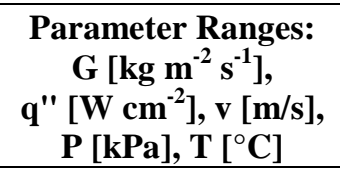 & Remarks \\
\hline $\begin{array}{l}\text { Bowers and Mudawar } \\
\text { (1994) [93] }\end{array}$ & $\begin{array}{l}\text { R113 in copper } \\
\text { circular }(\mathrm{N}=17) \\
\mathrm{D}=0.51 \text { and } 2.45\end{array}$ & $\begin{array}{l}\mathrm{q} "=0-200 \\
\Delta \mathrm{T}=10-32\end{array}$ & $\begin{array}{l}\text { Experiments on boiling and two-phase flow: } \\
\text { Boiling curves and two-phase flow data are obtained } \\
\text { Microchannels yield higher CHF }(28 \% \text { greater at } \mathrm{Q}=64 \mathrm{ml} / \mathrm{min}) \text { than minichannels, } \\
\text { with a larger } \triangle \mathrm{P}(30 \mathrm{kPa} \text { for micro, } 3 \mathrm{kPa} \text { for mini) }\end{array}$ \\
\hline $\begin{array}{l}\text { Celata et al. (1994) } \\
\text { [94] }\end{array}$ & $\begin{array}{l}\text { water in stainless steel } \\
\text { circular } \\
\mathrm{D}=2.5\end{array}$ & 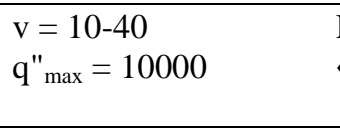 & $\begin{array}{l}\text { Experiments on subcooled boiling of water under high q": } \\
\text { - Experimental data do not match predictions from CHF correlations in the literature }\end{array}$ \\
\hline $\begin{array}{l}\text { Roach et al. (1999) } \\
\text { [95] }\end{array}$ & $\begin{array}{l}\text { water in copper } \\
\text { Circular and rod bundle } \\
\mathrm{D}_{\mathrm{h}}=1.131-1.45\end{array}$ & $\begin{array}{l}\mathrm{G}=250-1000 \\
\mathrm{x}_{\text {out }}=0.4-1.1 \\
\mathrm{P}=344-1204\end{array}$ & $\begin{array}{l}\text { Experiments on CHF in flow of subcooled water: } \\
\text { CHF is found to increase monotonically with increasing G or P } \\
\text { CHF depends on the channel cross-section geometry, and increases with increasing } \\
\mathrm{D}_{\mathrm{h}}\end{array}$ \\
\hline $\begin{array}{l}\text { Sturgis and Mudawar } \\
\text { (1999) [96] }\end{array}$ & $\begin{array}{l}\text { FC-72 in fiberglass } \\
\text { rectangular } \\
\mathrm{D}_{\mathrm{h}}=3.33\end{array}$ & $\begin{array}{l}\operatorname{Re}=2000-130000 \\
q^{\prime \prime}=0-105 \\
v=0.25-10\end{array}$ & $\begin{array}{l}\text { CHF experiments on long channels and flow visualization: } \\
\text { Propagation of vapor patches resembling a wavy vapor layer along the heated wall at } \\
\text { the critical heat flux are observed } \\
\text { Length and height of vapor patch is found to increase along the flow direction, and } \\
\text { decrease with increasing subcooling and velocity }\end{array}$ \\
\hline $\begin{array}{l}\text { Sturgis and Mudawar } \\
\text { (1999) [97] }\end{array}$ & $\begin{array}{l}\text { FC-72 in fiberglass } \\
\text { rectangular } \\
\mathrm{D}_{\mathrm{h}}=3.33\end{array}$ & $\begin{array}{l}\mathrm{Re}=2000-130000 \\
\mathrm{q}^{\prime}=0-105 \\
\mathrm{v}=0.25-10\end{array}$ & $\begin{array}{l}\text { Theoretical model for CHF data analysis: } \\
\text { Effect of periodic distribution of vapor patches is idealized as a sinusoidal interface } \\
\text { with amplitude and wavelength increasing in flow direction }\end{array}$ \\
\hline $\begin{array}{l}\text { Kosar and Peles } \\
(2007)[98]\end{array}$ & $\begin{array}{l}\text { R123 in silicon } \\
\text { rectangular }(\mathrm{N}=5) \\
\mathrm{D}_{\mathrm{h}}=0.223\end{array}$ & $\begin{array}{l}\mathrm{G}=291-1118 \\
\mathrm{q}=53-196 \\
\mathrm{P}=227-520\end{array}$ & $\begin{array}{l}\text { Experiments on CHF in parallel microchannels: } \\
\text { Dryout is the leading CHF mechanism } \\
\text { CHF increases with G and decreases with } \mathrm{x} \\
\text { CHF increases to a certain pressure, and decreases thereafter }\end{array}$ \\
\hline $\begin{array}{l}\text { Revellin and Thome } \\
\text { (2008) [99] }\end{array}$ & $\begin{array}{l}5 \text { fluids } \\
\text { circular and rectangular } \\
\mathrm{D}_{\mathrm{h}}=0.215-3.15\end{array}$ & $G=29-1600$ & $\begin{array}{l}\text { Development of a theoretical CHF model and comparison to database: } \\
-90 \% \text { of data can be predicted to within } \pm 20 \% \text { for several databases }\end{array}$ \\
\hline \multicolumn{4}{|c|}{ Flow Patterns and Flow Instabilities } \\
\hline $\begin{array}{l}\text { Ma and Peterson } \\
\text { (1996) [100] }\end{array}$ & V-shaped channel & & $\begin{array}{l}\text { Analysis of microgrooves with non-uniform heat input: } \\
\text { Analytical expression is developed for the evaporating film profile }\end{array}$ \\
\hline $\begin{array}{l}\text { Ha and Peterson } \\
\text { (1998) [101] }\end{array}$ & V-shaped channel & & $\begin{array}{l}\text { Analysis of axial flow of an evaporating thin film: } \\
\text { Perturbation method is used to solve the axial flow of an evaporating thin film } \\
\text { through a V-shaped microchannel with tilt }\end{array}$ \\
\hline
\end{tabular}




\begin{tabular}{|c|c|c|c|}
\hline Reference & $\begin{array}{l}\text { Fluid/Substrate, } \\
\text { Geometry, } D_{h}[\mathrm{~mm}], \mathbf{N}\end{array}$ & $\begin{array}{l}\text { Parameter Ranges: } \\
\text { G }\left[\mathrm{kg} \mathrm{m}^{-2} \mathrm{~s}^{-1}\right] \\
\mathbf{q}^{\prime \prime}\left[\mathbf{W} \mathbf{~ c m}^{-2}\right], \mathbf{v}[\mathbf{m} / \mathbf{s}] \\
\mathbf{P}[\mathbf{k P a}], \mathbf{T}\left[{ }^{\circ} \mathbf{C}\right]\end{array}$ & Remarks \\
\hline $\begin{array}{l}\text { Triplett et al. (1999) } \\
\text { [102] }\end{array}$ & $\begin{array}{l}\text { Air-water mixture in glass } \\
\text { circular and triangular } \\
\mathrm{D}_{\mathrm{h}}=1.1-1.5\end{array}$ & $\mathrm{v}=0.02-80$ & $\begin{array}{l}\text { Visual observation of flow patterns and pattern map generation: } \\
\text { Bubbly, churn, slug, slug-annular and annular flow patterns are observed }\end{array}$ \\
\hline $\begin{array}{l}\text { Jiang and Wong } \\
\text { (2001) [103] }\end{array}$ & $\begin{array}{l}\text { water in silicon } \\
\text { triangular }(\mathrm{N}=34-35) \\
\mathrm{D}_{\mathrm{h}}=26-53\end{array}$ & $\begin{array}{l}\mathrm{q}^{\prime \prime}=0.0-2.5 \\
\mathrm{v}=0.55-3.5 \mathrm{ml} / \mathrm{min}\end{array}$ & $\begin{array}{l}\text { Flow visualization in parallel triangular microchannels: } \\
\text { At low, medium and high q", a dominance of nucleation is replaced by unstable slug } \\
\text { flow, and thereafter by stable annular flow } \\
\text { Bubble flow mode is not observed }\end{array}$ \\
\hline $\begin{array}{l}\text { Nino et al. (2003) } \\
{[104]}\end{array}$ & $\begin{array}{l}\mathrm{R} 134 \mathrm{a} \text { in } \mathrm{PVC} \\
\text { rectangular }(\mathrm{N}=6) \\
\mathrm{D}_{\mathrm{h}}=1.5\end{array}$ & $\begin{array}{l}\mathrm{G}=50-300 \\
\mathrm{q}^{\prime \prime}=0 \\
\mathrm{x}=0.1-0.9\end{array}$ & $\begin{array}{l}\text { Flow visualization in parallel microchannels: } \\
\text { Several flow configurations may exist at the same time } \\
\text { Typical flow regime map does not apply } \\
\text { Probability of time fraction for a flow regime is proposed as alternative approach }\end{array}$ \\
\hline $\begin{array}{l}\text { Tu and Hrnjak (2003) } \\
{[105]}\end{array}$ & $\begin{array}{l}\mathrm{R} 134 \mathrm{a} \text { in PVC } \\
\text { rectangular } \\
\mathrm{D}_{\mathrm{h}}=0.168\end{array}$ & $\begin{array}{l}\mathrm{G}=279-461 \\
\mathrm{q}^{\prime \prime}=0 \\
\mathrm{x}=0.05-0.95\end{array}$ & $\begin{array}{l}\text { Pressure measurements and flow visualization in adiabatic flow: } \\
\text { Most of the existing correlations over-predict the pressure drop } \\
\text { Flow visualization shows that flow patterns in mini- and microchannels are similar } \\
\text { Slug/plug flow and annular flow regime dominate }\end{array}$ \\
\hline $\begin{array}{l}\text { Hollingsworth (2004) } \\
{[106]}\end{array}$ & $\begin{array}{l}\mathrm{R} 11 \text { in aluminum } \\
\text { rectangular } \\
\mathrm{D}_{\mathrm{h}}=0.98-3.6\end{array}$ & $\mathrm{~T}_{\mathrm{sat}}=24$ & $\begin{array}{l}\text { Imaging of flow boiling in minichannels: } \\
\text { Kandlikar (1990) correlation predicts h well } \\
\text { Boiling front can be identified with liquid crystal imaging }\end{array}$ \\
\hline $\begin{array}{l}\text { Molki et al. (2004) } \\
\text { [107] }\end{array}$ & $\begin{array}{l}\text { water in aluminum } \\
\text { transverse ribs } \\
\mathrm{D}_{\mathrm{h}}=1.93\end{array}$ & $\begin{array}{l}\mathrm{G}=100-225 \\
\mathrm{q}^{\prime \prime}=0.8-3.5 \\
\mathrm{~T}_{\mathrm{sat}}=15-30 \\
\mathrm{x}=0.0-0.9\end{array}$ & $\begin{array}{l}\text { Visualization, measurement, and modeling of refrigerant flow boiling: } \\
\text { regime } \\
\text { regime of the data were in the convective boiling regime, } 22 \% \text { in the nucleate boiling } \\
\text { Pressure increases linearly with } x\end{array}$ \\
\hline $\begin{array}{l}\text { Balsubramanian and } \\
\text { Kandlikar (2005) } \\
{[108]}\end{array}$ & $\begin{array}{l}\text { water in copper } \\
\text { rectangular }(\mathrm{N}=6) \\
\mathrm{D}_{\mathrm{h}}=0.33\end{array}$ & $\begin{array}{l}\mathrm{G}=120 \\
\mathrm{q}^{\prime \prime}=21-32\end{array}$ & $\begin{array}{l}\text { Experiments on flow patterns, pressure drop, and flow instabilities: } \\
\text { Dominant frequency of pressure drop fluctuations increases with increase in surface } \\
\text { temperature } \\
\text { Nucleate boiling is observed in the bulk liquid and a thin film surrounds the vapor } \\
\text { slugs } \\
\text { Slug flow is observed to be dominant for higher surface temperatures } \\
\text { Backflow causes flow maldistribution }\end{array}$ \\
\hline $\begin{array}{l}\text { Morgante and Fabre } \\
(2005) \text { [109] }\end{array}$ & $\begin{array}{l}\text { nitrogen and water } \\
\text { rectangular and circular } \\
\mathrm{D}_{\mathrm{h}}=1.0-4.0\end{array}$ & $\mathrm{q}^{\prime \prime}=0$ & $\begin{array}{l}\text { Flow pattern visualization in adiabatic two-phase flow: } \\
\text { Slug/plug flow and annular flow with smooth interface have been identified } \\
\text { Transition occurs at nearly constant critical gas velocity that depends on channel size } \\
\text { Surface tension and inertia can explain the observed transition }\end{array}$ \\
\hline
\end{tabular}




\begin{tabular}{|c|c|c|c|}
\hline Reference & $\begin{array}{l}\text { Fluid/Substrate, } \\
\text { Geometry, } \mathrm{D}_{\mathrm{h}}[\mathrm{mm}], \mathbf{N}\end{array}$ & 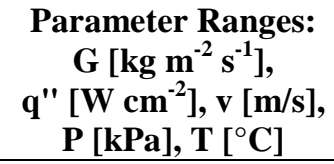 & Remarks \\
\hline $\begin{array}{l}\text { Lie and Lin (2006) } \\
\text { [110] }\end{array}$ & $\begin{array}{l}\mathrm{R} 134 \text { in copper } \\
\text { annular } \\
\mathrm{D}_{\mathrm{h}}=2.0-4.0\end{array}$ & $\begin{array}{l}\mathrm{Re}=200-300 \\
\mathrm{q}^{\prime \prime}=0-5.5 \\
\mathrm{~T}_{\text {sat }}=10-15\end{array}$ & $\begin{array}{l}\text { Flow measurement and visualization in a narrow annular duct: } \\
\text { Bubble diameter decreases as G increases due to higher shear force } \\
\text { A smaller gap leads to a higher frequency of bubbles } \\
\text { - At high q", the bubbles merge on the surface }\end{array}$ \\
\hline $\begin{array}{l}\text { Revellin et al. (2006) } \\
\text { [111] }\end{array}$ & $\begin{array}{l}\mathrm{R}-134 \mathrm{a} \text { in glass } \\
\text { circular } \\
\mathrm{D}_{\mathrm{h}}=0.5\end{array}$ & $\begin{array}{l}\mathrm{G}=350-2000 \\
\mathrm{q}^{\prime \prime}=0.4-12.9 \\
\mathrm{p}=770\end{array}$ & $\begin{array}{l}\text { Optical measurement of two-phase flow in microtubes: } \\
\text { Frequency of bubble generation and coalescence is determined } \\
\text { - Measured void fraction compares reasonably well to homogeneous values }\end{array}$ \\
\hline $\begin{array}{l}\text { Chang and Pan } \\
(2007)[112]\end{array}$ & $\begin{array}{l}\text { water } \\
\text { rectangular }(\mathrm{N}=15) \\
\mathrm{D}_{\mathrm{h}}=0.0863\end{array}$ & $\begin{array}{l}\mathrm{G}=22-110 \\
\mathrm{q}^{\prime \prime}=0-9\end{array}$ & $\begin{array}{l}\text { Investigation of flow instabilities in microchannels: } \\
\text { Magnitude of pressure fluctuation indicates flow reversal } \\
\text { Stable flow is observed only for outlet vapor qualities below and close to zero }\end{array}$ \\
\hline $\begin{array}{l}\text { Hardt et al. (2007) } \\
{[113]}\end{array}$ & $\begin{array}{l}\text { 2-propanol and water in } \\
\text { silicon } \\
\text { rectangular }(\mathrm{N}=16-300) \\
\mathrm{D}_{\mathrm{h}}=0.03-0.05\end{array}$ & $\begin{array}{l}\mathrm{G}=0-120 \\
\mathrm{v}=0-4\end{array}$ & $\begin{array}{l}\text { Experimental flow patterns and velocity trajectories during boiling in parallel } \\
\text { microchannels: } \\
\text { Film evaporation is dominant boiling regime } \\
\text { Parallel oscillations, chaotic oscillations and stationary vapor-liquid interfaces can } \\
\text { be found at different flow conditions }\end{array}$ \\
\hline $\begin{array}{l}\text { Hibiki et al. (2007) } \\
\text { [114] }\end{array}$ & $\begin{array}{l}\text { water and nitrogen } \\
\text { circular } \\
\mathrm{D}=1.02\end{array}$ & $\begin{array}{l}\mathrm{v}=1.02-4.89 \\
\mathrm{x}=0.01-0.25\end{array}$ & $\begin{array}{l}\text { Measurement of void fraction, interfacial area, gas velocity in vertical tube: } \\
\text { Drift flux model agrees well with measurements }\end{array}$ \\
\hline Hu et al. (2007) [115] & $\begin{array}{l}\text { water } \\
\text { rectangular } \\
\mathrm{D}_{\mathrm{h}}=0.104\end{array}$ & $\begin{array}{l}\mathrm{G}=170,360 \\
\mathrm{q}^{\prime \prime}=20-53\end{array}$ & $\begin{array}{l}\text { Investigation of flow boiling instabilities in microchannels: } \\
\text { Fluctuations of P and G correspond to transition between bubbly flow and semi- } \\
\text { annular flow } \\
\text { Time period of cyclic behavior is very long }(100-200 \mathrm{~s})\end{array}$ \\
\hline $\begin{array}{l}\text { Kuan and Kandlikar } \\
\text { (2007) [116] }\end{array}$ & $\begin{array}{l}\text { water in copper } \\
\text { rectangular }(\mathrm{N}=6) \\
\mathrm{D}_{\mathrm{h}}=0.332\end{array}$ & $\begin{array}{l}\mathrm{G}=144-388 \\
\mathrm{q}^{\prime \prime}=15-40 \\
\mathrm{P}=100\end{array}$ & $\begin{array}{l}\text { Experiments on effect of flow stabilization using flow restrictors and machined } \\
\text { nucleation sites: } \\
\text { Flow restrictors increased pressure drop considerably } \\
\text { Altogether inlet orifices did not improve heat transfer performance }\end{array}$ \\
\hline $\begin{array}{l}\text { Kuo and Peles (2007) } \\
{[117]}\end{array}$ & $\begin{array}{l}\text { water in silicon } \\
\text { rectangular }(\mathrm{N}=5) \\
\mathrm{D}_{\mathrm{h}}=0.223\end{array}$ & $\begin{array}{l}\mathrm{G}=83-303 \\
\mathrm{q}^{\prime \prime}=0-64.3\end{array}$ & $\begin{array}{l}\text { Measurements on microchannels with reentrant cavities: } \\
\text { Nucleation is uniform and reproducible } \\
\text { CHF is enhanced }\end{array}$ \\
\hline Qi et al. (2007) [118] & $\begin{array}{l}\text { nitrogen in stainless steel } \\
\text { circular } \\
\mathrm{D}_{\mathrm{h}}=0.53-1.93\end{array}$ & $\begin{array}{l}\mathrm{G}=440-3000 \\
\mathrm{q}^{\prime \prime}=5.1-21.4 \\
\mathrm{P}=140-890\end{array}$ & $\begin{array}{l}\text { Measurement of ONB, flow instability and pressure drop: } \\
\text { Long-period ( } 50-60 \mathrm{~s}) \text { periodic flow oscillations are observed } \\
\text { Homogeneous model predicts pressure drop well }\end{array}$ \\
\hline $\begin{array}{l}\text { Revellin and Thome } \\
\text { (2007) [119] }\end{array}$ & $\begin{array}{l}\text { R-134a, R245fa in glass } \\
\text { circular } \\
\mathrm{D}=0.5,0.8\end{array}$ & $\begin{array}{l}\mathrm{T}_{\text {sat }}=26,30,35 \\
\mathrm{G}=200-2000 \\
\mathrm{q}^{\prime \prime}=0.32-42.2\end{array}$ & $\begin{array}{l}\text { Optical measurement of two-phase flow in microtubes: } \\
\text { New flow map for small tubes presented } \\
\text { No significant difference between } 0.5 \text { and } 0.8 \mathrm{~mm} \text { tube }\end{array}$ \\
\hline
\end{tabular}




\begin{tabular}{|c|c|c|c|}
\hline Reference & $\begin{array}{l}\text { Fluid/Substrate, } \\
\text { Geometry, } D_{\mathrm{h}}[\mathrm{mm}], \mathrm{N}\end{array}$ & 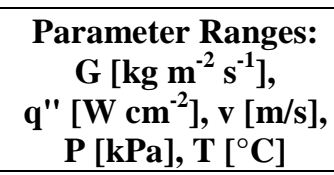 & Remarks \\
\hline $\begin{array}{l}\text { Wang et al. (2007) } \\
{[120]}\end{array}$ & $\begin{array}{l}\text { water in silicon } \\
\text { trapezoidal }(\mathrm{N}=1-8) \\
\mathrm{D}_{\mathrm{h}}=0.186\end{array}$ & $\begin{array}{l}\mathrm{G}=150-500 \\
\mathrm{q}^{\prime \prime}=10-60\end{array}$ & $\begin{array}{l}\text { Investigation of flow boiling instabilities: } \\
\text { Three boiling modes in single and parallel microchannels are possible: a) stable flow, } \\
\text { b) unstable flow with long-period oscillations, and c) unstable flow with short-period } \\
\text { oscillations }\end{array}$ \\
\hline $\begin{array}{l}\text { Alm et al. (2008) } \\
\text { [121] }\end{array}$ & $\begin{array}{l}\text { water in ceramic } \\
\text { rectangular }(\mathrm{N}=34-51) \\
\mathrm{D}_{\mathrm{h}}=0.28-0.313\end{array}$ & $\mathrm{M}=20-120 \mathrm{~kg} / \mathrm{h}$ & $\begin{array}{l}\text { Measurement of pressure loss and heat transfer in microchannel heat exchanger: } \\
\text { Channel blockages from the joining process of the heat exchanger account for } \\
\text { differences between theory and experiment }\end{array}$ \\
\hline $\begin{array}{l}\text { Revellin et al. (2008) } \\
{[122]}\end{array}$ & $\begin{array}{l}\text { R-134a } \\
\text { circular } \\
\mathrm{D}=0.5\end{array}$ & $\begin{array}{l}\mathrm{G}=323-1986 \\
\mathrm{~T}_{\mathrm{sat}}=26-35\end{array}$ & $\begin{array}{l}\text { Measurement of bubble length and velocity in microchannels: } \\
\text { Bubble velocity is proportional to bubble length }\end{array}$ \\
\hline $\begin{array}{l}\text { Wang (2008 in press) } \\
\text { [123] }\end{array}$ & $\begin{array}{l}\text { water in silicon } \\
\text { trapezoidal }(\mathrm{N}=8) \\
\mathrm{D}_{\mathrm{h}}=0.186\end{array}$ & $\begin{array}{l}\mathrm{G}=150-500 \\
\mathrm{q}^{\prime \prime}=10-60\end{array}$ & $\begin{array}{l}\text { Investigation of flow boiling instabilities: } \\
\text { Exit vapor quality can be used to classify stable and unstable flow conditions } \\
\text { Inlet and outlet plenum configuration greatly affects stability } \\
\text { With inlet flow restrictors, stable flow can be achieved }\end{array}$ \\
\hline
\end{tabular}


Table 2. Studies in the literature from which correlations are selected, and their ranges of applicability.

\begin{tabular}{|c|c|c|}
\hline Reference & $\begin{array}{c}\text { Fluid, } \\
\text { Channel Geometry }\end{array}$ & 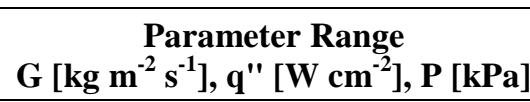 \\
\hline $\begin{array}{l}\text { Edelstein et al. (1984) [124] } \\
\text { Chen (1966) 125] }\end{array}$ & $\begin{array}{l}\text { Water, Methanol, Pentane, Heptane, } \\
\text { Benzene,... }\end{array}$ & $\begin{array}{l}\mathrm{P}=55-3500 \\
\mathrm{q}^{\prime \prime}=0.6-240 \\
\mathrm{x}=0.01-0.71\end{array}$ \\
\hline $\begin{array}{l}\text { Bennett and Chen (1980) [126] } \\
\text { Bennet et al. (1980) [127] }\end{array}$ & $\begin{array}{l}\text { Water, Methanol, Pentane, Heptane, } \\
\text { Benzene,... }\end{array}$ & $\begin{array}{l}P=55-3500 \\
q^{\prime \prime}=0.6-240 \\
X=0.01-0.71\end{array}$ \\
\hline $\begin{array}{l}\text { Shah (1982) } \\
{[128]}\end{array}$ & $\begin{array}{l}\mathrm{R} 11, \mathrm{R} 12, \mathrm{R} 22, \mathrm{R} 502, \ldots \\
\mathrm{D}_{\mathrm{h}}=5.0-15.8 \mathrm{~mm}\end{array}$ & $\begin{array}{l}\mathrm{G}=70-11071 \\
\mathrm{q}^{\prime \prime}=9-121.5 \\
\mathrm{x}=0.0-0.7\end{array}$ \\
\hline $\begin{array}{l}\text { Lazarek and Black (1982) } \\
\text { [26] }\end{array}$ & $\begin{array}{l}\mathrm{R} 113 \\
\mathrm{D}_{\mathrm{h}}=3.1 \mathrm{~mm}\end{array}$ & $\begin{array}{l}\mathrm{G}=125-750 \\
\mathrm{q}^{\prime \prime}=1.4-38 \\
\mathrm{x}=0.0-0.6\end{array}$ \\
\hline $\begin{array}{l}\text { Gungor and Winterton (1986) } \\
\text { [129] }\end{array}$ & $\begin{array}{l}\text { Water, R11, R12, R113,... } \\
\mathrm{D}_{\mathrm{h}}=2.95-32.0 \mathrm{~mm}\end{array}$ & $\begin{array}{l}\mathrm{G}=67-61518 \\
\mathrm{q}=0.11-228 \\
\mathrm{x}=0.0-1.0\end{array}$ \\
\hline $\begin{array}{l}\text { Kandlikar }(1990,1991) \\
{[130,131]}\end{array}$ & $\begin{array}{l}\text { Water, R11, R12, R22, R113, } \\
\text { Nitrogen,... } \\
\mathrm{D}_{\mathrm{h}}=4.6-32 \mathrm{~mm}\end{array}$ & $\begin{array}{l}\mathrm{G}=13-8179 \\
\mathrm{q}^{\prime \prime}=0.03-228 \\
\mathrm{x}=0.00-0.99\end{array}$ \\
\hline $\begin{array}{l}\text { Liu and Winterton (1991) } \\
\text { [132] }\end{array}$ & $\begin{array}{l}\text { Water and refrigerants } \\
\mathrm{D}_{\mathrm{h}}=2.95-32.0 \mathrm{~mm}\end{array}$ & $\begin{array}{l}\mathrm{G}=12.4-8157 \\
\mathrm{q}^{\prime \prime}=0.35-262 \\
\mathrm{x}=0.0-0.95\end{array}$ \\
\hline $\begin{array}{l}\text { Steiner and Taborek (1992) } \\
\text { [133] }\end{array}$ & $\begin{array}{l}\text { Water, refrigerants, cryogenics } \\
D_{h}=1-32 \mathrm{~mm}\end{array}$ & $\begin{array}{l}\mathrm{G}=3.9-4850 \\
\mathrm{q}=0.08-460 \\
\mathrm{P}=10-11000 \\
\mathrm{x}=0.0-1.0\end{array}$ \\
\hline $\begin{array}{l}\text { Tran et al. (1996) } \\
\text { [27] }\end{array}$ & $\begin{array}{l}\mathrm{R} 12, \mathrm{R} 113 \\
\mathrm{D}_{\mathrm{h}}=2.4-2.92 \mathrm{~mm}\end{array}$ & $\begin{array}{l}\mathrm{G}=44-832 \\
\mathrm{q}=0.36-12.9 \\
\mathrm{P}=510-820 \\
\mathrm{x}=0-0.94\end{array}$ \\
\hline $\begin{array}{l}\text { Yan and Lin (1998) } \\
\text { [28] }\end{array}$ & $\begin{array}{l}\mathrm{R} 134 \mathrm{a} \\
\mathrm{D}_{\mathrm{h}}=2.0 \mathrm{~mm}\end{array}$ & $\begin{array}{l}\mathrm{G}=50-200 \\
\mathrm{q}^{\prime \prime}=0.4-1.9 \\
\mathrm{x}=0.0-0.9\end{array}$ \\
\hline Lee and Lee (2001) [25] & $\begin{array}{l}\mathrm{R} 113 \\
\mathrm{D}_{\mathrm{h}}=0.78-3.6 \mathrm{~mm}\end{array}$ & $\begin{array}{l}\mathrm{G}=50-200 \\
\mathrm{q}^{\prime \prime}=0-1.5 \\
\mathrm{x}=0.15-0.75\end{array}$ \\
\hline $\begin{array}{l}\text { Warrier et al. (2002) } \\
\text { [52] }\end{array}$ & $\begin{array}{l}\text { FC- } 84 \\
D_{h}=0.75 \mathrm{~mm}\end{array}$ & $\begin{array}{l}\mathrm{G}=557-1600 \\
\mathrm{q}^{\prime \prime}=0-5.99 \\
\mathrm{x}=0.03-0.55\end{array}$ \\
\hline $\begin{array}{l}\text { Yu et al. (2002) } \\
\text { [53] }\end{array}$ & $\begin{array}{l}\text { Water } \\
\mathrm{D}_{\mathrm{h}}=2.98 \mathrm{~mm}\end{array}$ & $\begin{array}{l}\mathrm{G}=50-200 \\
\mathrm{P}=200 \\
\mathrm{x}=0-0.9\end{array}$ \\
\hline $\begin{array}{l}\text { Haynes and Fletcher (2003) } \\
{[134]}\end{array}$ & $\begin{array}{l}\mathrm{R} 11, \mathrm{R} 123 \\
\mathrm{D}_{\mathrm{h}}=0.92-1.95 \mathrm{~mm}\end{array}$ & $\begin{array}{l}\mathrm{G}=110-1840 \\
\mathrm{q}^{\prime \prime}=1.1-17.0 \\
\mathrm{x}=0.0-1.0\end{array}$ \\
\hline $\begin{array}{l}\text { Sumith et al. (2003) } \\
\text { [58] }\end{array}$ & $\begin{array}{l}\text { Water } \\
\mathrm{D}_{\mathrm{h}}=1.45 \mathrm{~mm}\end{array}$ & $\begin{array}{l}\mathrm{G}=23.4-152.7 \\
\mathrm{q}=1-71.5 \\
\mathrm{x}=0.0-0.7\end{array}$ \\
\hline
\end{tabular}




\begin{tabular}{|c|c|c|}
\hline Reference & $\begin{array}{c}\text { Fluid, } \\
\text { Channel Geometry }\end{array}$ & $\begin{array}{c}\text { Parameter Range } \\
\mathrm{G}\left[\mathrm{kg} \mathrm{m}^{-2} \mathrm{~s}^{-1}\right], \mathrm{q}^{\prime \prime}\left[\mathrm{W} \mathrm{cm}^{-2}\right], \mathrm{P}[\mathrm{kPa}]\end{array}$ \\
\hline $\begin{array}{l}\text { Balasubramanian and Kandlikar } \\
\text { (2004) } \\
\text { [135] }\end{array}$ & $\begin{array}{l}\text { Water, R113, R123, R141b,... } \\
D_{h}=0.19-2.92 \mathrm{~mm}\end{array}$ & $\begin{array}{l}\mathrm{G}=50-570 \\
\mathrm{q}^{\prime \prime}=0.5-9.1 \\
\mathrm{x}=0.00-0.98\end{array}$ \\
\hline $\begin{array}{l}\text { Thome et al. (2004) } \\
{[62,63]}\end{array}$ & $\begin{array}{l}\mathrm{R} 11, \mathrm{R} 12, \mathrm{R} 113, \mathrm{R} 123, \mathrm{R} 134 \mathrm{a}, \\
\mathrm{R} 141 \mathrm{~b}, \mathrm{CO}_{2} \\
\mathrm{D}_{\mathrm{h}}=0.7-3.1 \mathrm{~mm}\end{array}$ & $\begin{array}{l}\mathrm{G}=50-502 \\
\mathrm{q}=0.5-17.8 \\
\mathrm{x}=0.01-0.99\end{array}$ \\
\hline $\begin{array}{l}\text { Lee and Mudawar (2005) } \\
\text { [89] }\end{array}$ & $\begin{array}{l}\text { R134a, water } \\
D_{h}=0.35 \mathrm{~mm}\end{array}$ & $\begin{array}{l}\mathrm{G}=127-654 \\
\mathrm{q}^{\prime \prime}=15.9-93.8 \\
\mathrm{x}=0.26-0.87\end{array}$ \\
\hline $\begin{array}{l}\text { Zhang et al. (2005) } \\
\text { [68] }\end{array}$ & $\begin{array}{l}\text { Water, R11, R12, and R113 } \\
\mathrm{D}_{\mathrm{h}}=0.78-6.0 \mathrm{~mm}\end{array}$ & $\begin{array}{l}\mathrm{G}=23.4-560 \\
\mathrm{q}^{\prime \prime}=0.3-80.3\end{array}$ \\
\hline $\begin{array}{l}\text { Yun et al. (2006) } \\
{[33,136]}\end{array}$ & $\begin{array}{l}\mathrm{R} 410 \mathrm{~A} \\
\mathrm{D}_{\mathrm{h}}=1.36,1.44 \mathrm{~mm}\end{array}$ & $\begin{array}{l}\mathrm{G}=200-400 \\
\mathrm{q}=1-2 \\
\mathrm{x}=0.1-0.85\end{array}$ \\
\hline $\begin{array}{l}\text { Liu and Garimella (2007) } \\
\text { [77] }\end{array}$ & $\begin{array}{l}\text { Water; } \\
\mathrm{D}_{\mathrm{h}}=0.38,0.59 \mathrm{~mm}\end{array}$ & $\begin{array}{l}\mathrm{G}=221-283 \\
\mathrm{q}=0-129 \\
\mathrm{x}=0-0.2\end{array}$ \\
\hline $\begin{array}{l}\text { Saitoh et al. (2007) } \\
{[82]}\end{array}$ & $\begin{array}{l}\mathrm{R} 134 \mathrm{a} \\
\mathrm{D}_{\mathrm{h}}=0.5-11.0 \mathrm{~mm}\end{array}$ & \\
\hline $\begin{array}{l}\text { Lee and Garimella (2008) } \\
\text { [85] }\end{array}$ & $\begin{array}{l}\text { Water } \\
\mathrm{D}_{\mathrm{h}}=0.16-0.57 \mathrm{~mm}\end{array}$ & $\begin{array}{l}\mathrm{G}=218-935 \\
\mathrm{q}=0-80 \\
\mathrm{x}=0.0-0.2\end{array}$ \\
\hline $\begin{array}{l}\text { Cooper (pool boiling) (1984) } \\
\text { [137] }\end{array}$ & $\begin{array}{l}\text { Water, refrigerants, organic fluids, } \\
\text { cryogens }\end{array}$ & $\mathrm{q}^{\prime \prime}=0.01-60$ \\
\hline $\begin{array}{l}\text { Gorenflo (pool boiling) (1993) } \\
\text { [138] }\end{array}$ & $\begin{array}{l}\text { Several refrigerants, water and } \\
\text { cryogenics }\end{array}$ & $\mathrm{q}^{\prime \prime}=0-30$ \\
\hline
\end{tabular}


Table 3. Database of studies from which heat transfer coefficients in minichannels and microchannels are obtained for comparison against the correlations assessed in this

review.

\begin{tabular}{|c|c|c|c|}
\hline Reference & $\begin{array}{c}\text { Fluid, substrate, orientation, } \\
\text { and geometry }\end{array}$ & 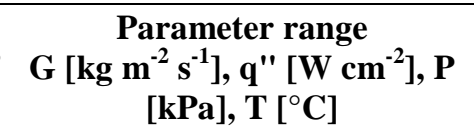 & Data \\
\hline Yan and Lin [28] & $\begin{array}{l}\mathrm{R}-134 \mathrm{a} \text { in copper } \\
\text { circular }(\mathrm{N}=28) \\
\text { horizontal } \\
\mathrm{D}=2.0 \mathrm{~mm}\end{array}$ & $\begin{array}{l}\mathrm{G}=50-200 \\
\mathrm{q}=0.5-2.0 \\
\mathrm{~T}_{\text {sat }}=5-31 \\
\mathrm{x}=0.05-0.85\end{array}$ & $\begin{array}{l}\text { from paper; } \\
106 \text { points }\end{array}$ \\
\hline Bao et al. [32] & $\begin{array}{l}\mathrm{R} 11, \mathrm{R} 123 \text { in copper } \\
\text { circular }(\mathrm{N}=1) \\
\text { horizontal } \\
\mathrm{D}=1.95\end{array}$ & $\begin{array}{l}G=50-1800 \\
q=0.5-20 \\
P=200-500 \\
x=0.0-0.9\end{array}$ & $\begin{array}{l}\text { from paper; } \\
100 \text { points }\end{array}$ \\
\hline Lin et al. [29] & $\begin{array}{l}\text { Water and } \mathrm{R}-141 \mathrm{~b} \\
\text { circular }(\mathrm{N}=1) \\
\text { horizontal } \\
\mathrm{D}=1 \mathrm{~mm}\end{array}$ & $\begin{array}{l}\mathrm{G}=510 \\
\mathrm{q}=1-115 \\
\mathrm{~T}_{\text {sat }}=39-56 \\
\mathrm{x}=0-1\end{array}$ & $\begin{array}{l}\text { from paper; } \\
133 \text { points }\end{array}$ \\
\hline $\begin{array}{l}\text { Agostini et al. } \\
\text { [31] }\end{array}$ & $\begin{array}{l}\text { R-134a in aluminum } \\
\text { rectangular }(\mathrm{N}=11,18) \\
\text { vertical } \\
D_{\mathrm{h}}=0.77,2.01 \mathrm{~mm}\end{array}$ & $\begin{array}{l}\mathrm{G}=83-467 \\
\mathrm{q}=0.44-1.5 \\
\mathrm{P}=405-517 \\
\mathrm{x}=0.0-1.0\end{array}$ & $\begin{array}{l}\text { from paper; } \\
207 \text { points }\end{array}$ \\
\hline Saitoh et al. [82] & $\begin{array}{l}\mathrm{R}-134 \mathrm{a} \\
\text { circular }(\mathrm{N}=1) \\
\text { horizontal } \\
\mathrm{D}=0.51,1.12 \mathrm{~mm}\end{array}$ & $\begin{array}{l}\mathrm{G}=150-450 \\
\mathrm{q}=0.5-3.9 \\
\mathrm{~T}_{\text {sat }}=5-15 \\
\mathrm{x}=0.0-1.0\end{array}$ & $\begin{array}{l}\text { from paper; } \\
281 \text { points }\end{array}$ \\
\hline Yun et al. [33] & $\begin{array}{l}\mathrm{R} 410 \mathrm{~A} \text { in aluminum } \\
\text { rectangular }(\mathrm{N}=7,8) \\
\text { horizontal } \\
\mathrm{D}_{\mathrm{h}}=1.36,1.44 \mathrm{~mm}\end{array}$ & $\begin{array}{l}\mathrm{G}=200-400 \\
\mathrm{q}=1-2 \\
\mathrm{~T}_{\text {sat }}=0,5,10 \\
\mathrm{x}=0.0-0.85\end{array}$ & $\begin{array}{l}\text { from paper; } \\
164 \text { points }\end{array}$ \\
\hline Qi et al. [79] & $\begin{array}{l}\text { Nitrogen in stainless } \\
\text { circular }(\mathrm{N}=1) \\
\text { vertical } \\
\mathrm{D}=0.53-1.93 \mathrm{~mm}\end{array}$ & $\begin{array}{l}\mathrm{G}=440-3000 \\
\mathrm{q}=5-21 \\
\mathrm{~T}_{\text {sat }}=-194 \\
\mathrm{x}=0-0.9\end{array}$ & $\begin{array}{l}\text { from paper; } \\
184 \text { points }\end{array}$ \\
\hline $\begin{array}{l}\text { Harirchian and } \\
\text { Garimella [74] }\end{array}$ & $\begin{array}{l}\text { FC-77 in silicon } \\
\text { rectangular }(\mathrm{N}=2-60) \\
\text { horizontal } \\
\mathrm{D}_{\mathrm{h}}=0.16-0.57 \mathrm{~mm} \\
\end{array}$ & $\begin{array}{l}\mathrm{G}=250-1600 \\
\mathrm{q}^{\prime \prime}=0-30 \\
\mathrm{~T}_{\text {sat }}=90-93 \\
\mathrm{x}=0-1\end{array}$ & $\begin{array}{l}\text { original data set; } \\
327 \text { points }\end{array}$ \\
\hline $\begin{array}{l}\text { Chen and } \\
\text { Garimella [69, } \\
\text { 73] }\end{array}$ & $\begin{array}{l}\text { FC-77 in copper and silicon } \\
\text { rectangular }(\mathrm{N}=10,24) \\
\text { horizontal } \\
D_{\mathrm{h}}=0.84,0.39 \mathrm{~mm}\end{array}$ & $\begin{array}{l}\mathrm{G}=63.5-440 \\
\mathrm{q}^{\prime \prime}=2-80 \\
\mathrm{~T}_{\text {sat }}=97 \\
\mathrm{x}=0.01-0.99\end{array}$ & $\begin{array}{l}\text { original data set } \\
115 \text { points }\end{array}$ \\
\hline $\begin{array}{l}\text { Bertsch et al. } \\
{[30]}\end{array}$ & $\begin{array}{l}\mathrm{R} 134 \mathrm{a} \text { in copper } \\
\text { rectangular }(\mathrm{N}=17,33) \\
\text { horizontal } \\
\mathrm{D}_{\mathrm{h}}=1.089,0.544 \mathrm{~mm} \\
\end{array}$ & $\begin{array}{l}\mathrm{G}=20-170 \\
\mathrm{q}=5-21 \mathrm{~W} \\
\mathrm{~T}_{\text {sat }}=8.9,18.7,29 \\
\mathrm{x}=0-0.95\end{array}$ & $\begin{array}{l}\text { original data set; } \\
230 \text { points }\end{array}$ \\
\hline$\overline{\text { Total }}$ & $\begin{array}{l}8 \text { fluids } \\
D_{h}=0.16-2.01 \mathrm{~mm}\end{array}$ & $\begin{array}{l}G=20-3000 \\
q=0.4-115 \\
T_{\text {sat }}=-194-97 \\
x=0-1\end{array}$ & $\begin{array}{l}10 \text { data sets } \\
1847 \text { points }\end{array}$ \\
\hline
\end{tabular}


Table 4. Mean relative error (MRE), mean absolute error (MAE), and the percentage of predictions which fall within $\pm 30 \%$ of the measurements from each data set.

\begin{tabular}{|c|c|c|c|c|c|c|c|c|c|c|c|}
\hline $\begin{array}{c}\text { Database } \rightarrow \\
\downarrow \text { Correlation }\end{array}$ & $\begin{array}{c}\text { Yan and Lin } \\
{[28]}\end{array}$ & $\begin{array}{c}\text { Bao et al. } \\
\text { [32] }\end{array}$ & $\begin{array}{l}\text { Lin et al. } \\
\text { [29] }\end{array}$ & $\begin{array}{l}\text { Agostini et } \\
\text { al. [31] }\end{array}$ & $\begin{array}{l}\text { Saitoh et al. } \\
\text { [82] }\end{array}$ & $\begin{array}{c}\text { Yun et al. } \\
\text { [33] }\end{array}$ & Qi et al. [7ك & $\begin{array}{c}\text { Harirchian } \\
\text { 19] and Garimella } \\
\text { [74] }\end{array}$ & $\begin{array}{c}\text { Chen and } \\
\text { Garimella } \\
{[69,73]}\end{array}$ & $\begin{array}{c}\text { Bertsch et al. } \\
{[30]}\end{array}$ & $\begin{array}{c}\text { Average } \\
\text { over all } \\
\text { data sets }\end{array}$ \\
\hline \multirow{3}{*}{$\begin{array}{l}\text { Edelstein et al., } \\
\text { Chen }[124,125]\end{array}$} & 24.6 & -37.7 & 224.0 & -39.6 & 42.8 & -55.0 & 103.6 & 161.1 & 14.3 & 28.7 & 46.7 \\
\hline & 35.9 & 37.7 & 224.0 & 59.0 & 43.3 & 55.0 & 109.7 & 161.1 & 70.3 & 40.5 & 83.7 \\
\hline & 60.6 & 27.0 & 2.3 & 11.9 & 38.7 & 3.3 & 27.6 & 0.0 & 2.0 & 54.4 & 22.8 \\
\hline \multirow{3}{*}{$\begin{array}{l}\text { Bennett and } \\
\text { Chen }[126,127]\end{array}$} & 26.4 & -36.5 & 277.1 & -35.4 & 64.7 & -49.6 & 64.9 & 186.3 & 10.2 & 10.2 & 51.8 \\
\hline & 36.9 & 36.5 & 277.1 & 53.7 & 64.7 & 49.6 & 73.8 & 186.3 & 66.7 & 30.5 & 87.6 \\
\hline & 47.7 & 29.4 & 2.3 & 17.1 & 11.3 & 8.8 & 35.4 & 1.6 & 3.1 & 64.3 & 22.1 \\
\hline \multirow{3}{*}{ Shah [128] } & 15.6 & 38.4 & 878.3 & -38.9 & 249.4 & 5.6 & 98.9 & 836.3 & 143.2 & 17.2 & 224.4 \\
\hline & 35.9 & 43.2 & 878.3 & 48.0 & 249.4 & 24.6 & 100.5 & 836.3 & 143.3 & 39.0 & 239.9 \\
\hline & 45.9 & 43.6 & 0.0 & 17.6 & 2.8 & 65.9 & 14.9 & 0.0 & 6.1 & 48.9 & 24.6 \\
\hline \multirow{3}{*}{$\begin{array}{l}\text { Lazarek and } \\
\text { Black [26] }\end{array}$} & -27.7 & -4.3 & 64.4 & -56.3 & -22.5 & -63.6 & 189.0 & 149.6 & 17.5 & 29.6 & 27.6 \\
\hline & 31.9 & 8.2 & 64.4 & 57.7 & 23.8 & 63.6 & 189.0 & 149.6 & 38.6 & 35.4 & 66.2 \\
\hline & 42.2 & 100.0 & 8.0 & 10.9 & 70.8 & 0.0 & 1.7 & 0.0 & 60.2 & 48.9 & 34.3 \\
\hline \multirow{3}{*}{$\begin{array}{l}\text { Gungor and } \\
\text { Winterton [129] }\end{array}$} & 35.2 & 32.0 & 247.5 & -13.5 & 59.0 & -37.8 & 151.2 & 484.1 & 273.9 & 136.8 & 136.8 \\
\hline & 36.0 & 32.1 & 247.5 & 34.7 & 59.0 & 37.8 & 151.3 & 484.1 & 273.9 & 136.8 & 149.3 \\
\hline & 52.3 & 46.0 & 0.0 & 46.1 & 17.0 & 28.6 & 5.5 & 0.0 & 0.0 & 3.3 & 19.9 \\
\hline \multirow{3}{*}{$\begin{array}{l}\text { Kandlikar [130, } \\
\text { 131] }\end{array}$} & -62.2 & -37.6 & 6.0 & -44.9 & -19.3 & -72.5 & 91.8 & 102.2 & 80.5 & 126.9 & 17.1 \\
\hline & 62.2 & 38.1 & 13.1 & 48.3 & 25.7 & 72.5 & 92.8 & 106.7 & 102.5 & 126.9 & 68.9 \\
\hline & 1.8 & 41.1 & 97.7 & 23.3 & 67.9 & 0.0 & 22.1 & 29.7 & 45.9 & 6.6 & 33.6 \\
\hline \multirow{3}{*}{$\begin{array}{l}\text { Liu and } \\
\text { Winterton [132] }\end{array}$} & -27.4 & -31.4 & 128.3 & -58.1 & -16.2 & -67.8 & 24.3 & 79.0 & -35.7 & -9.3 & -1.4 \\
\hline & 30.7 & 31.4 & 128.3 & 58.6 & 19.6 & 67.8 & 37.3 & 79.0 & 44.6 & 27.0 & 52.4 \\
\hline & 50.5 & 36.8 & 5.7 & 9.3 & 69.8 & 0.0 & 50.3 & 28.1 & 29.6 & 69.2 & 34.9 \\
\hline \multirow{3}{*}{$\begin{array}{l}\text { Steiner and } \\
\text { Taborek [133] }\end{array}$} & 63.8 & 49.7 & 203.3 & 9.2 & 80.4 & -41.8 & 188.6 & 148.1 & 22.2 & 262.5 & 98.6 \\
\hline & 64.5 & 49.7 & 203.3 & 35.6 & 80.8 & 41.8 & 188.6 & 148.1 & 41.5 & 262.5 & 111.6 \\
\hline & 22.0 & 0.0 & 0.0 & 62.2 & 18.9 & 20.9 & 5.0 & 0.0 & 62.2 & 0.0 & 19.1 \\
\hline \multirow{3}{*}{ Tran et al. [27] } & -14.8 & -13.1 & -27.1 & -62.2 & -53.8 & -77.3 & -27.9 & 61.4 & 22.6 & -15.1 & -20.7 \\
\hline & 26.2 & 13.2 & 27.1 & 62.6 & 53.8 & 77.3 & 31.7 & 61.4 & 78.4 & 26.3 & 45.8 \\
\hline & 67.0 & 100.0 & 64.8 & 7.3 & 15.1 & 0.0 & 49.2 & 14.4 & 0.0 & 57.7 & 37.5 \\
\hline
\end{tabular}

${ }^{*}$ The three numbers for each correlation represent MRE, MAE, and percentage of predictions which fall within $\pm 30 \%$ of the measurements, respectively. In order not to extrapolate the correlations beyond their suggested range, the vapor quality range was limited to $70 \%$. Numbers in bold indicate MAE below $30 \%$, or more than half the data predicted within $\pm 30 \%$ of the measurement. 


\begin{tabular}{|c|c|c|c|c|c|c|c|c|c|c|c|}
\hline $\begin{array}{c}\text { Database } \rightarrow \\
\downarrow \text { Correlation }\end{array}$ & $\begin{array}{c}\text { Yan and Lin } \\
{[28]}\end{array}$ & $\begin{array}{c}\text { Bao et al. } \\
\text { [32] }\end{array}$ & $\begin{array}{l}\text { Lin et al. } \\
\text { [29] }\end{array}$ & $\begin{array}{l}\text { Agostini et } \\
\text { al. [31] }\end{array}$ & $\begin{array}{l}\text { Saitoh et al. } \\
\text { [82] }\end{array}$ & $\begin{array}{l}\text { Yun et al. } \\
\text { [33] }\end{array}$ & Qi et al. [79] & $\begin{array}{c}\text { Harirchian } \\
\text { ] and Garimella } \\
\text { [74] }\end{array}$ & $\begin{array}{c}\text { Chen and } \\
\text { Garimella } \\
{[69,73]}\end{array}$ & $\begin{array}{c}\text { Bertsch et al. } \\
{[30]}\end{array}$ & $\begin{array}{c}\text { Average } \\
\text { over all } \\
\text { data sets }\end{array}$ \\
\hline \multirow{3}{*}{$\begin{array}{l}\text { Yan and Lin } \\
{[28]}\end{array}$} & -39.4 & 25.6 & -6.2 & -61.8 & 365.1 & 75.5 & 30924.6 & 16247.0 & -20.4 & -16.9 & 4749.3 \\
\hline & 63.7 & 67.1 & 48.0 & 61.8 & 392.6 & 127.2 & 30943.8 & 16248.6 & 61.6 & 87.2 & 4810.2 \\
\hline & 8.3 & 35.0 & 30.7 & 9.3 & 13.2 & 5.5 & 9.4 & 2.6 & 15.3 & 12.1 & 14.1 \\
\hline \multirow{3}{*}{ Lee and Lee [25] } & 12.9 & -56.9 & 203.8 & 27.1 & 139.3 & -42.2 & 23.8 & 275.3 & 82.0 & 113.6 & 77.9 \\
\hline & 28.7 & 56.9 & 203.8 & 43.4 & 144.5 & 42.2 & 51.8 & 295.4 & 123.7 & 114.6 & 110.5 \\
\hline & 56.9 & 2.5 & 1.1 & 61.7 & 17.0 & 12.1 & 47.0 & 18.2 & 16.3 & 18.7 & 25.1 \\
\hline \multirow{3}{*}{$\begin{array}{l}\text { Warrier et al. } \\
{[52]}\end{array}$} & -81.8 & -90.0 & -54.0 & -74.8 & -71.9 & -94.5 & -87.0 & 25.1 & 35.0 & 10.9 & -48.3 \\
\hline & 81.8 & 90.0 & 54.2 & 74.8 & 71.9 & 94.5 & 87.0 & 68.1 & 78.8 & 41.3 & 74.2 \\
\hline & 0.0 & 0.0 & 13.6 & 1.6 & 0.9 & 0.0 & 0.0 & 27.2 & 29.6 & 50.5 & 12.3 \\
\hline \multirow{3}{*}{ Yu et al. [53] } & 1911.3 & 1930.7 & 2204.4 & 916.1 & 1140.0 & 423.8 & 1465.9 & 4131.5 & 3146.8 & 1866.9 & 1913.7 \\
\hline & 1911.3 & 1930.7 & 2204.4 & 916.1 & 1140.0 & 423.8 & 1465.9 & 4131.5 & 3146.8 & 1866.9 & 1913.7 \\
\hline & 0.0 & 0.0 & 0.0 & 0.0 & 0.0 & 0.0 & 0.0 & 0.0 & 0.0 & 0.0 & 0.0 \\
\hline \multirow{3}{*}{$\begin{array}{l}\text { Haynes and } \\
\text { Fletcher [134] }\end{array}$} & -8.4 & 14.6 & 29.3 & -50.2 & -29.4 & -65.0 & -6.8 & 126.6 & 50.8 & 45.6 & 10.7 \\
\hline & 24.3 & 14.6 & 29.9 & 52.1 & 29.4 & 65.0 & 22.0 & 126.6 & 61.1 & 47.7 & 47.3 \\
\hline & 72.5 & 98.8 & 47.7 & 12.4 & 50.9 & 0.0 & 69.1 & 0.6 & 63.3 & 33.5 & 44.9 \\
\hline \multirow{3}{*}{$\begin{array}{l}\text { Sumith et al. } \\
\text { [58] }\end{array}$} & -37.2 & -65.5 & 147.0 & -48.8 & 23.4 & -59.8 & 24.9 & 82.3 & -39.7 & -38.6 & -1.2 \\
\hline & 38.8 & 65.5 & 149.6 & 49.6 & 30.9 & 59.8 & 54.0 & 101.1 & 58.8 & 45.8 & 65.4 \\
\hline & 40.4 & 1.2 & 12.5 & 15.5 & 63.2 & 0.0 & 46.4 & 35.1 & 14.3 & 27.5 & 25.6 \\
\hline \multirow{3}{*}{$\begin{array}{l}\text { Balasubrama- } \\
\text { nian and } \\
\text { Kandlikar [135] }\end{array}$} & -55.3 & -39.5 & 6.0 & -44.9 & -18.8 & -75.6 & 89.1 & 99.0 & 80.5 & 126.8 & 16.7 \\
\hline & 55.3 & 40.0 & 13.1 & 48.3 & 25.2 & 75.6 & 90.1 & 103.1 & 102.5 & 126.8 & 68.0 \\
\hline & 1.8 & 38.7 & 97.7 & 23.3 & 67.9 & 0.0 & 22.1 & 29.7 & 45.9 & 6.6 & 33.4 \\
\hline \multirow{3}{*}{$\begin{array}{l}\text { Thome et al. [62, } \\
63]\end{array}$} & 25.3 & 4.6 & -1.3 & -33.4 & -29.4 & -44.5 & -53.7 & -38.9 & -43.7 & 86.7 & -12.8 \\
\hline & 33.6 & 11.4 & 18.6 & 41.5 & 33.1 & 44.8 & 55.2 & 43.4 & 59.4 & 87.7 & 42.9 \\
\hline & 63.3 & 93.9 & 83.0 & 23.8 & 39.6 & 17.6 & 13.8 & 39.3 & 24.5 & 18.1 & 41.7 \\
\hline \multirow{3}{*}{$\begin{array}{l}\text { Lee and } \\
\text { Mudawar [89] }\end{array}$} & -73.1 & -79.9 & -39.6 & -65.7 & -55.8 & -90.1 & -53.0 & 447.3 & 97.5 & 12.1 & 10.0 \\
\hline & 73.1 & 79.9 & 41.4 & 65.7 & 64.1 & 90.1 & 54.6 & 461.2 & 139.0 & 45.8 & 111.5 \\
\hline & 5.5 & 0.0 & 30.7 & 1.0 & 5.7 & 0.0 & 19.9 & 6.1 & 27.6 & 44.0 & 14.0 \\
\hline \multirow{3}{*}{ Zhang et al. [68] } & -4.2 & -58.5 & 73.0 & -51.6 & -15.6 & -80.9 & 53.9 & 100.2 & 11.1 & 35.3 & 6.3 \\
\hline & 36.9 & 58.5 & 76.4 & 67.0 & 22.7 & 80.9 & 80.4 & 100.2 & 79.4 & 47.0 & 64.9 \\
\hline & 46.8 & 0.6 & 19.3 & 8.3 & 76.4 & 0.0 & 34.3 & 11.8 & 0.0 & 52.7 & 25.0 \\
\hline \multirow{3}{*}{$\begin{array}{l}\text { Yun et al. [33, } \\
136]\end{array}$} & 274.0 & 123.2 & 457.6 & 142.0 & 197.3 & -4.4 & 44.8 & 505.2 & 354.2 & 177.1 & 227.1 \\
\hline & 274.0 & 123.2 & 457.6 & 142.0 & 197.3 & 16.9 & 49.4 & 505.2 & 354.2 & 177.1 & 229.7 \\
\hline & 0.0 & 0.0 & 0.0 & 10.4 & 0.0 & 93.4 & 48.6 & 0.0 & 0.0 & 0.0 & 15.2 \\
\hline \multirow{3}{*}{$\begin{array}{l}\text { Liu and } \\
\text { Garimella [77] }\end{array}$} & -17.3 & -77.7 & 79.9 & -59.0 & -37.8 & -77.4 & -70.1 & 81.1 & -31.2 & -40.4 & -25.0 \\
\hline & 33.1 & 77.9 & 81.6 & 59.0 & 39.0 & 77.4 & 71.0 & 83.7 & 55.2 & 60.4 & 63.8 \\
\hline & 45.0 & 3.7 & 29.5 & 9.3 & 49.1 & 0.0 & 1.1 & 31.9 & 6.1 & 10.4 & 18.6 \\
\hline
\end{tabular}




\begin{tabular}{|c|c|c|c|c|c|c|c|c|c|c|c|}
\hline $\begin{array}{c}\text { Database } \rightarrow \\
\downarrow \text { Correlation }\end{array}$ & $\begin{array}{c}\text { Yan and Lin } \\
{[28]}\end{array}$ & $\begin{array}{c}\text { Bao et al. } \\
{[32]}\end{array}$ & $\begin{array}{l}\text { Lin et al. } \\
\text { [29] }\end{array}$ & $\begin{array}{l}\text { Agostini et } \\
\text { al. [31] }\end{array}$ & $\begin{array}{c}\text { Saitoh et al. } \\
\text { [82] }\end{array}$ & $\begin{array}{c}\text { Yun et al. } \\
\text { [33] }\end{array}$ & Qi et al. [79 & $\begin{array}{c}\text { Harirchian } \\
\text { 9] and Garimella } \\
\text { [74] }\end{array}$ & $\begin{array}{c}\text { Chen and } \\
\text { Garimella } \\
{[69,73]}\end{array}$ & $\begin{array}{c}\text { Bertsch et al. } \\
\text { [30] }\end{array}$ & $\begin{array}{c}\text { Average } \\
\text { over all } \\
\text { data sets }\end{array}$ \\
\hline \multirow{3}{*}{ Saitoh et al. [82] } & -6.1 & -11.9 & 123.9 & -49.5 & -0.6 & -58.9 & 44.8 & 211.7 & 81.3 & 30.1 & 36.5 \\
\hline & 19.9 & 12.8 & 123.9 & 52.8 & 12.0 & 58.9 & 54.0 & 211.7 & 82.5 & 35.1 & 66.4 \\
\hline & 80.7 & 99.4 & 4.5 & 12.4 & 90.6 & 0.0 & 44.8 & 0.0 & 59.2 & 50.5 & 44.2 \\
\hline \multirow{3}{*}{$\begin{array}{l}\text { Lee and } \\
\text { Garimella [85] }\end{array}$} & 1167.0 & 930.8 & 951.1 & 452.6 & 607.9 & 217.3 & 1435.9 & 339.9 & 217.7 & 1116.0 & 743.6 \\
\hline & 1167.0 & 930.8 & 951.1 & 452.6 & 607.9 & 217.3 & 1435.9 & 339.9 & 217.7 & 1116.0 & 743.6 \\
\hline & 0.0 & 0.0 & 0.0 & 0.0 & 0.0 & 0.0 & 0.0 & 0.0 & 2.0 & 0.0 & 0.2 \\
\hline \multirow{3}{*}{$\begin{array}{l}\text { Cooper (pool } \\
\text { boiling) [137] }\end{array}$} & -26.1 & -23.6 & 18.0 & -57.9 & -41.5 & -69.8 & 27.8 & 1.1 & -35.6 & 14.4 & -19.3 \\
\hline & 32.1 & 23.6 & 20.5 & 58.9 & 41.5 & 69.8 & 37.9 & 7.3 & 46.1 & 23.0 & $\underline{36.1^{\S}}$ \\
\hline & 43.1 & 92.6 & 75.0 & 9.8 & 10.4 & 0.0 & 49.7 & 100.0 & 28.6 & 72.0 & 48.1 \\
\hline \multirow{3}{*}{$\begin{array}{l}\text { Gorenflo (pool } \\
\text { boiling) [138] }\end{array}$} & -10.7 & 14.0 & 30.2 & -54.3 & -35.6 & -67.8 & -12.7 & 154.0 & 57.1 & 46.2 & $\overline{12.1}$ \\
\hline & 26.5 & 14.0 & 31.3 & 56.0 & 35.6 & 67.8 & 25.7 & 154.0 & 64.4 & 49.1 & 52.4 \\
\hline & 64.2 & 98.8 & 44.3 & 10.9 & 32.1 & 0.0 & 60.8 & 0.0 & 63.3 & 34.1 & 40.8 \\
\hline
\end{tabular}

\footnotetext{
${ }^{\S}$ Best overall performance with respect to MAE and amount of data represented within $\pm 30 \%$
} 

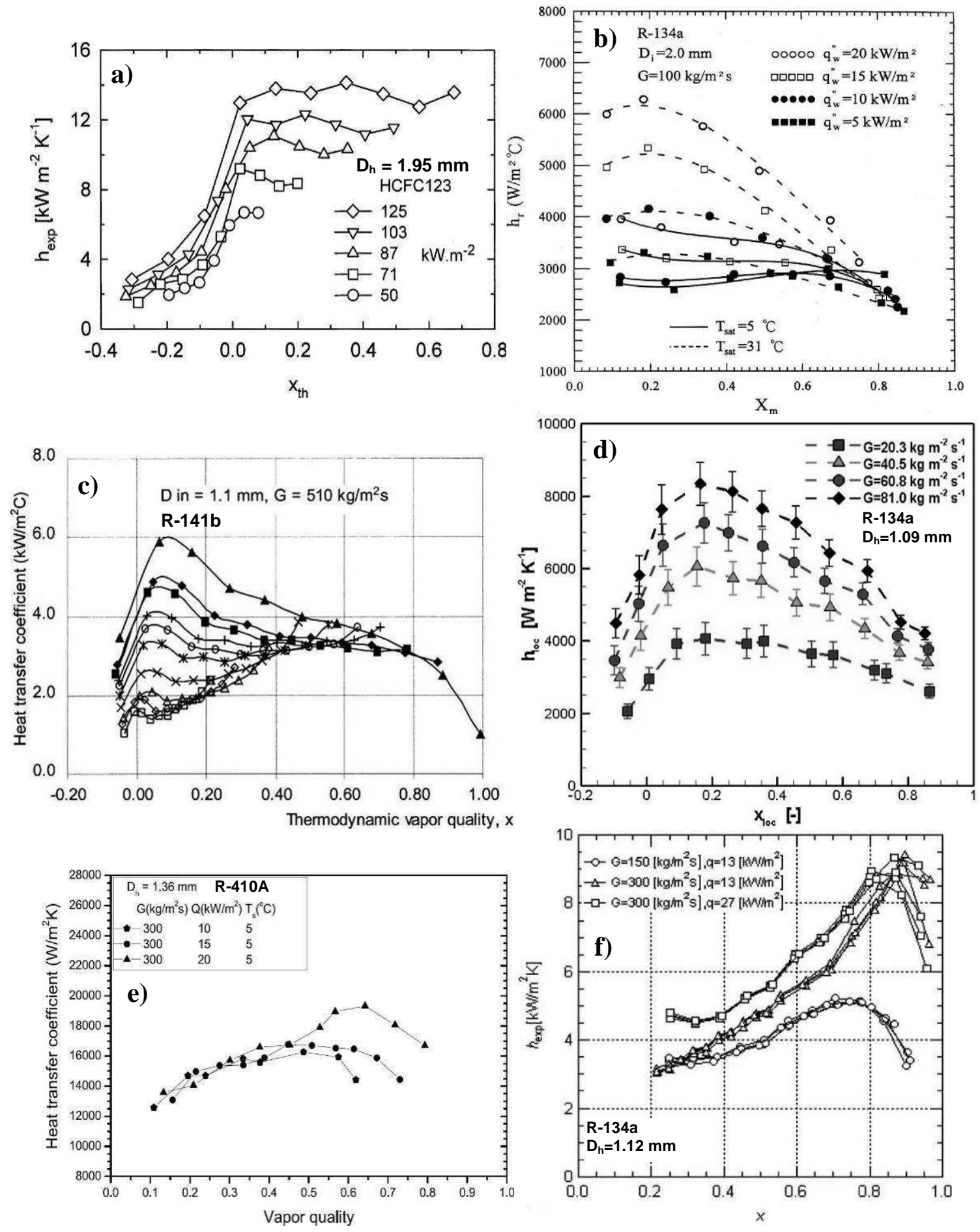

Figure 1. Variation of heat transfer coefficient with thermodynamic vapor quality from the studies of: (a) Bao et al. [32], (b) Yan and Lin [28], (c) Lin et al. [29], (d) Bertsch et al. [30], (e) Yun et al. [33], and (f) Saitoh et al. [24].

** Permission to reproduce results from the original sources to be obtained. 

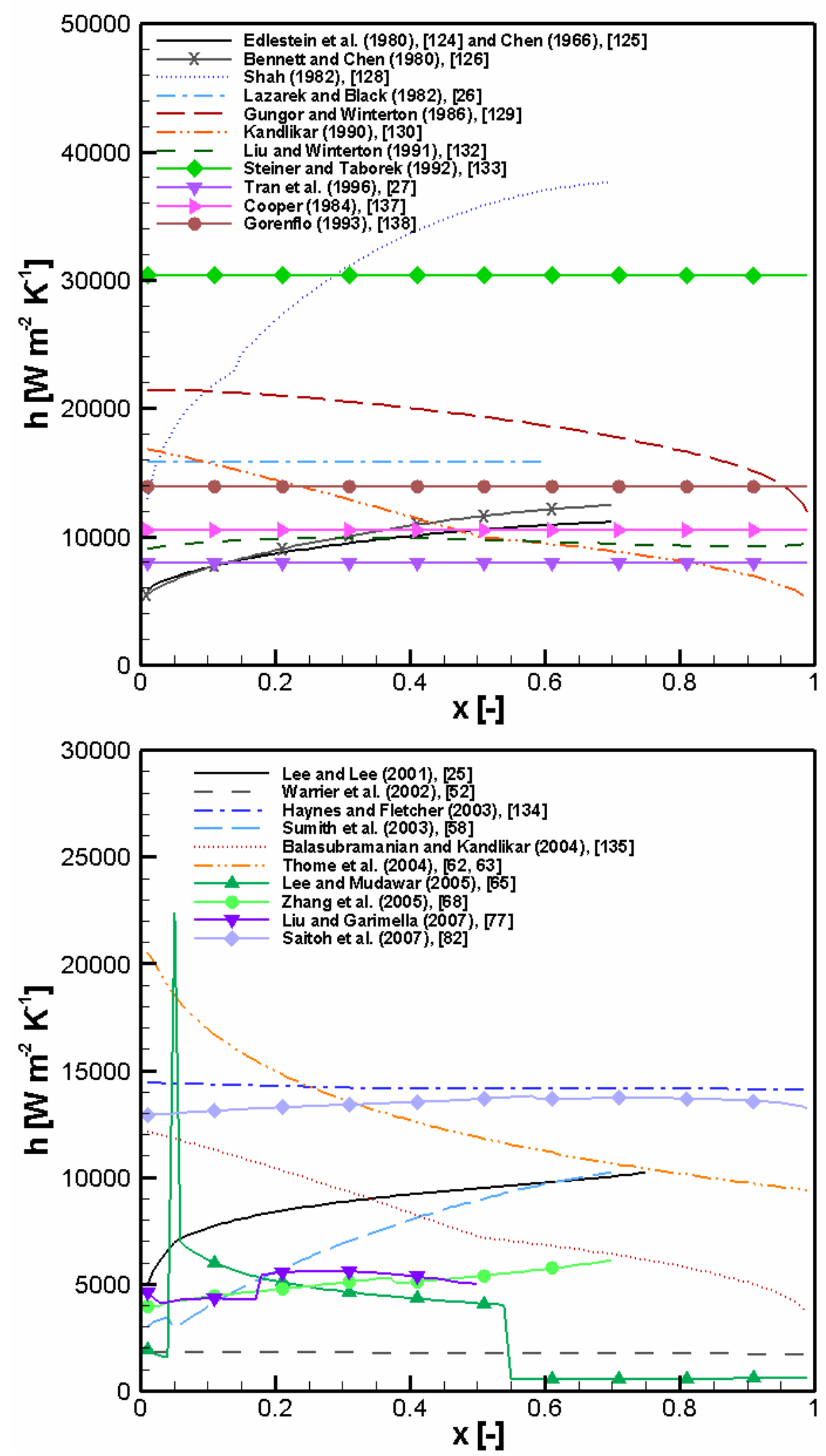

Figure 2. Variation of heat transfer coefficient with thermodynamic vapor quality as calculated for R134a in copper at $\mathrm{G}=500 \mathrm{~kg} \mathrm{~m}^{-2} \mathrm{~s}^{-1}, \mathrm{q}^{\prime \prime}=10 \mathrm{~W} \mathrm{~cm}{ }^{-2}$ and $\mathrm{D}=1 \mathrm{~mm}$ from flow boiling and pool boiling correlations for conventional-sized channels (top), and minichannels and microchannels (bottom). 

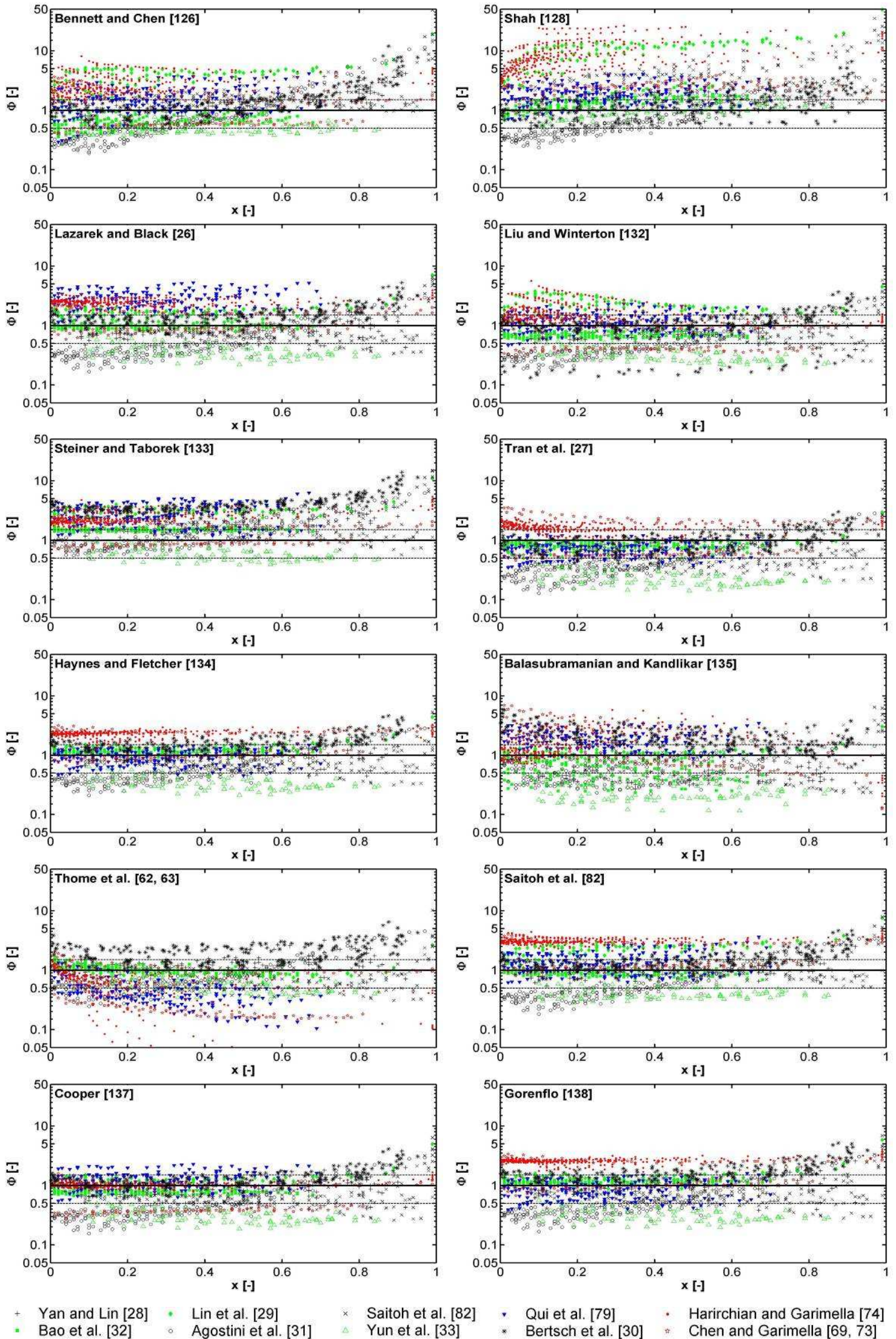

Figure 3. Ratio of calculated to measured heat transfer coefficient $(\Phi)$ as a function of thermodynamic quality using a number of correlations in the literature. 

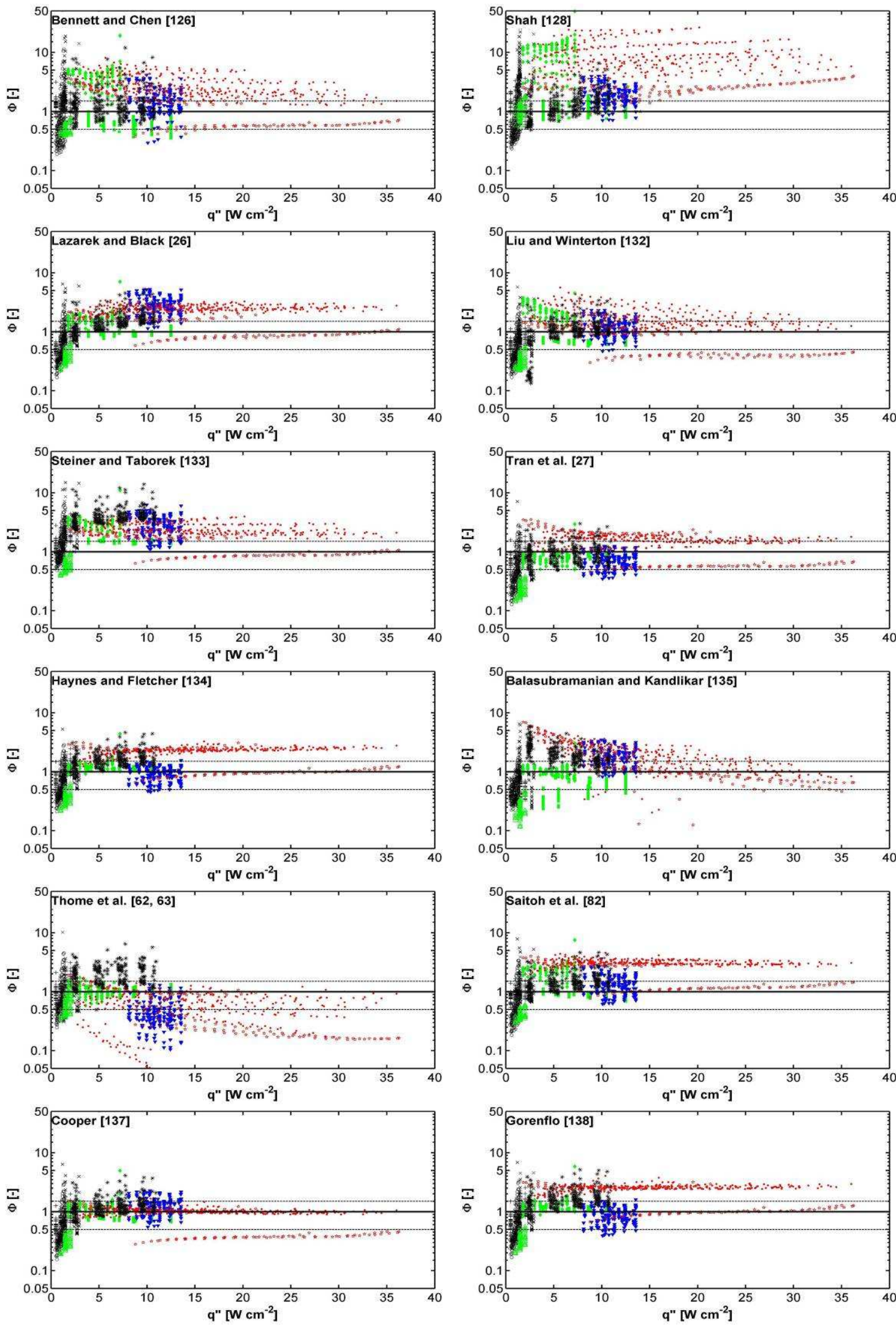

+ Yan and Lin [28] * Lin et al. [29] $\quad \times$ Saitoh et al. [82] * Qui et al. [79] * Harirchian and Garimella [74]

Figure 4. Ratio of calculated to measured heat transfer coefficient $(\Phi)$ as a function of heat flux using a number of correlations in the literature. 

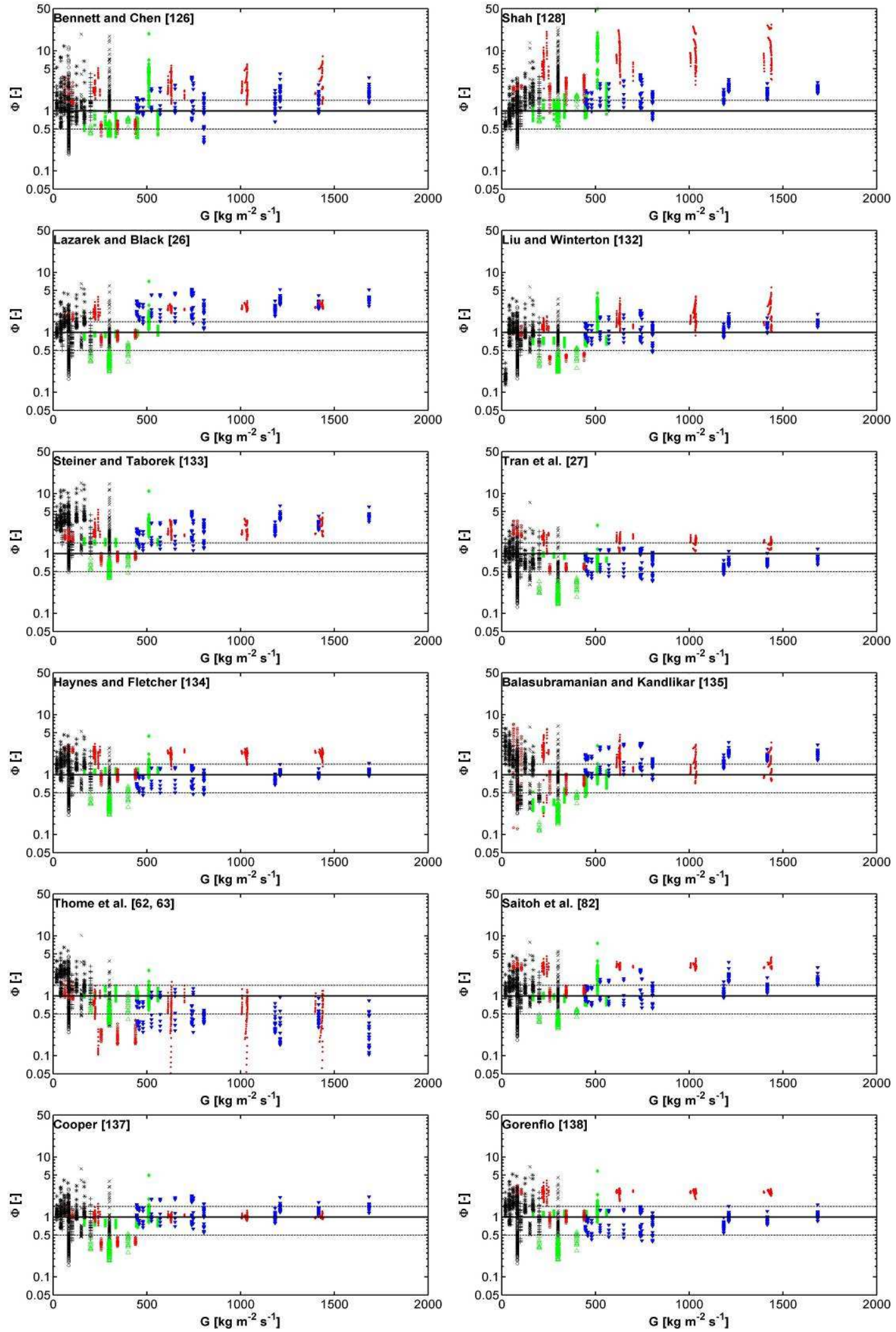

$+\quad$ Yan and Lin [28] * Lin et al. [29] $\quad \times \quad$ Saitoh et al. [82] * Qui et al. [79] * Harirchian and Garimella [74]

Bao et al. [32] * Agostini et al. [31] $\triangle$ Yun et al. [33] * Bertsch et al. [30] * Chen and Garimella [69, 73]

Figure 5. Ratio of calculated to measured heat transfer coefficient $(\Phi)$ as a function of mass flux using a number of correlations in the literature. 


\section{References}

[1] C. B. Sobhan, and S.V. Garimella, 2001, "A comparative analysis of studies on heat transfer and fluid flow in microchannels," Microscale Thermophysical Engineering, 5, pp. $293-311$.

[2] A. Bar-Cohen, and E. Rahim, 2007, "Modeling and prediction of two-phase refrigerant flow regimes and heat transfer characteristics in microgap channels," in proceedings of the 5th International Conference on Nanochannels, Microchannels and Minichannels, ICNMM2007.

[3] J. R. Thome, 2004, "Boiling in microchannels: a review of experiment and theory," Int. J. Heat and Fluid Flow, 25, pp.128-139.

[4] S.V. Garimella, V. Singhal and D. Liu, 2006, "On-chip thermal management with microchannel heat sinks and integrated micropumps," Proceedings of the IEEE, 94 (8), pp.1534-1548.

[5] G. Hetsroni, D. Klein, A. Mosyak, Z. Segal, and E. Pogrebnyak, 2004, "Convective boiling in parallel microchannels," Microscale Thermophysical Engineering, 8, pp. 403-421.

[6] S. V. Garimella, 2006, "Advances in mesoscale thermal management technologies for microelectronics," Microelectronics Journal, 37 (11), pp.11651185.

[7] B. Agostini, M. Fabbri, J. E. Park, L. Wojtan, J.R. Thome and B. Michel, 2007, "State of the art of high heat flux cooling technologies," Heat Transfer Engineering, 28 (4), pp. 258-281.

[8] S. V. Garimella, and C. B. Sobhan, 2003, "Transport in microchannels - a critical review," Annual Reviews in Heat Transfer, 13, pp. 1-50.

[9] S. G. Kandlikar and W. J. Grande, 2003, "Evolution of microchannel flow passages - thermohydraulic performance and fabrication technology," Heat Transfer Engineering, 24 (1), pp. 3-17.

[10] S. G. Kandlikar, 2002, "Fundamental issues related to flow boiling in minichannels and microchannels," Experimental Thermal and Fluid Science, 26, pp. 389-407.

[11] A. E. Bergles, S. G. Kandlikar, 2003, "Critical heat flux in microchannels: Experimental issues and guidelines for measurement," in proceedings of 1st Int. Conf. on Microchannels and Minichannels, ICMM2003-1016. 
[12] A. E. Bergles, J. H. Lienhard, G. E. Kendall and P. Griffith, 2003, "Boiling and evaporation in small diameter channels, "Heat Transfer Engineering, 24 (1), pp. $18-40$.

[13] S. G. Kandlikar, 2006, "Nucleaton characteristics and stability considerations during flow boiling in microchannels," Experimental Thermal and Fluid Science, 30, pp. 441-447.

[14] J. R. Thome, 2006, "State-of-the-art overview of boiling and two-phase flows in microchannels," Heat Transfer Engineering, 27 (9), pp. 4-19.

[15] V. Dupont and J. R. Thome, 2005, "Evaporation in microchannels: influence of the channel diameter on heat transfer," Microfluid Nanofluid, 1, pp.119-127.

[16] S. G. Kandlikar and M. E. Steinke, 2003, "Predicting heat transfer during flow boiling in minichannels and microchannels," ASHRAE Transactions, 109 (1), CH-03-13-1.

[17] C. Vlasie, H. Macchi, J. Guilpart and B. Agostini, 2004, "Flow boiling in small diameter channels,” Int. J. Refrigeration, 27, pp. 191-201.

[18] R. R. Riehl, P. Seleghim and J. M. Ochterbeck, 1998, "Comparison of heat transfer correlations for single- and two-phase microchannel flows for miroelectronics cooling," in proceedings of InterSociety Conf. on Thermal Phenomena, pp. 409-416.

[19] G. Ribatski, L. Wojtan and J. R. Thome, 2006, "An analysis of experimental data and prediction methods for two-phase frictional pressure drop and flow boiling heat transfer in micro-scale channels," Experimental Thermal and Fluid Science, 31, pp. 1-12.

[20] D. Shiferaw, X. Huo, T. G. Karayiannis and D. B. R. Kenning, 2007, "Examination of heat transfer correlations and a model for flow boiling of R134a in small diameter tubes," Int. J. Heat Mass Transfer, 50, pp. 5177-5193.

[21] S. Trutassanawin, E.A. Groll, S.V. Garimella and L. Cremaschi, 2006, "Experimental investigation of a miniature-scale refrigeration system for electronics cooling," IEEE Transactions Components and Packaging Technologies, 29, pp. $678-687$.

[22] R. Mongia, K. Masahiro, and E. DiStefano, 2006, "Small scale refrigeration system for electronics cooling within a notebook computer," Proceedings of ITHERM'06. 
[23] P. E. Phelan, J. Swanson, F. Chiriac and V. Chiriac, 2004, "Designing a mesoscale vapor compression refrigerator for Cooling high-power microelectronics," Proceedings of ITHERM'04.

[24] S. Saitoh, H. Daiguji, and E. Hihara, 2005, "Effect of tube diameter on boiling heat transfer of R-134a in horizontal small-diameter tubes," Int. J. Heat and Mass Transfer, 48, pp. 4473-4984.

[25] H. J. Lee and S. Y. Lee, 2001, "Heat transfer correlation for boiling flows in small rectangular horizontal channels with low aspect ratios," Int. J. Multiphase Flow, 27, pp. 2043-2062.

[26] G. M. Lazarek and S. H. Black, 1982, "Evaporative heat transfer, pressure drop and critical heat flux in a small vertical tube with R-113," Int. J. Heat Mass Transfer, 25 (7), pp. 945-960.

[27] T. N. Tran, M. W. Wambsganss and D. M. France, 1996, "Small circular and rectangular-channel boiling with two refrigerants," Int. J. Multiphase Flow, 22, pp. 485-498.

[28] Y. Yan and T. Lin, 1998, "Evaporation heat transfer and pressure drop of refrigerant R-134a in a small pipe,” Int. J. Heat Mass Transfer, 41, pp. 4183-4194.

[29] S. Lin, P. A. Kew and K. Cornwell, 2001, "Two-phase heat transfer to a refrigerant in a 1mm diameter tube," Int. J. Refrigeration, 24, pp. 51-56.

[30] S. S. Bertsch, E. A. Groll and S. V. Garimella, 2008, "Refrigerant flow boiling heat transfer in parallel microchannels as a function of local vapor quality," Int. J. Heat Mass Transfer, in press.

[31] B. Agostini, A. Bontemps, B. Watel and B. Thonon, 2003, "Boiling heat transfer in mini-channels: Influence of the hydraulic diameter," in proceedings of International Congress of Refrigeration 2003, ICR0070.

[32] Z. Y. Bao, D. F. Fletcher and B. S. Haynes, 2000, "Flow boiling heat transfer of freon R11 and HCFC123 in narrow passages," Int. J. Heat Mass Transfer, 43, pp. 3347-3358.

[33] R. Yun, J. H. Heo and Y. Kim, 2006, "Evaporative heat transfer and pressure drop of R410A in microchannels," Int. J. Refrigeration, 29, pp. 92-100.

[34] W. Qu and I. Mudawar, 2003, "Flow boiling heat transfer in two-phase microchannel heat sinks - I. Experimental investigation and assessment of correlation methods," Int. J. Heat Mass Transfer, 46, pp. 2755-2771. 
[35] M. E. Steinke and S. G. Kandlikar, 2004, "An experimental investigation of flow boiling characteristics of water in parallel microchannels," J. Heat Transfer, 126, pp. 518-526.

[36] S. A. Klein, 1992-2007, "Engineering Equation Solver," F-Chart Software, www.fChart.com.

[37] E. W. Lemmon, M. O. McLinden and M. L Huber, 2002, "REFPROP 7, Reference Fluid Thermodynamic and Transport Properties," NIST Standard Reference Database 23, Version 7.0.

[38] 3M Specialty Materials, 2000, "Fluorinert Electronic Liquid FC-77 - Product Information," 3M Center, Building 223-6S-04, St. Paul, MN 55144.

[39] X. F. Peng and B. X. Wang, 1993, "Forced convection and flow boiling heat transfer for liquid flowing through microchannels," Int. J. Heat Mass Transfer, 36, pp. 3421-3427.

[40] M. W. Wambsganss, D. M. France, J. A. Jendrzejcyk and T. N. Tran, 1993, "Boiling heat transfer in a horizontal small-diameter tube," J. Heat Transfer, 115, pp. 963-972.

[41] X. F. Peng, B. X. Wang, G. P. Peterson and H. B. Ma, 1995, "Experimental investigation of heat transfer in flat plates with rectangular microchannels," Int. J. Heat Mass Transfer, 38, pp. 127-137.

[42] J. M. Cuta, C. E. McDonald and A. Shekarriz, 1996, "Forced convection heat transfer in parallel channel array microchannel heat exchanger," Advances in Energy Efficiency, Heat/Mass Transfer Enhancement, ASME PID-Vol. 2/HTD, Vol. 338, pp. 17-23.

[43] R. Mertz, A. Wein and M. Groll, 1996, "Experimental investigation of flow boiling heat transfer in narrow channels," Heat and Technology, 14, pp. 47-54.

[44] X. F. Peng, G. P. Peterson and B. X. Wang, 1996, "Flow boiling of binary mixtures in microchannel plates," Int. J. Heat Mass Transfer, 39, pp. 1257-1264.

[45] P. A. Kew and K. Cornwell, 1997, "Correlations for the prediction of boiling heat transfer in small-diameter channels," Applied Thermal Engineering, 17 (8-10), pp. 705-715.

[46] X. F. Peng and B. X. Wang, 1998, "Forced-convection and boiling characteristics in microchannels," in proceedings of Heat Transfer - $11^{\text {th }}$ International Heat Transfer Conference, Vol. 1, pp. 371-390. 
[47] X. F. Peng, H. Y. Hu and B. X. Wang, 1998, "Flow boiling through V-shape microchannels," Experimental Heat Transfer, 11, pp. 87-90.

[48] X. F. Peng H. Y. Hu and B. X. Wang, 1998, "Boiling nucleation during liquid flow in microchannels," Int. J. Heat Mass Transfer, 41, pp. 101-106.

[49] T. S. Ravigururajan, 1998, "Impact of channel geometry on two-phase flow heat transfer characteristics of refrigerants in microchannel heat exchangers," J. Heat Transfer, 120, pp. 485-491.

[50] Y. Zhao, M. Molki, M. M. Ohadi and S. V. Dessiatoun, 2000, "Flow boiling of $\mathrm{CO}_{2}$ in microchannels," ASHRAE Transactions: Symposia, DA-00-2-1, pp. 437445.

[51] A. M. Jacobi and J. R. Thome, 2002, "Heat transfer model for evaporation of elongated bubble flows in microchannels," J. of Heat Transfer, 124, pp. 11311136.

[52] G. R. Warrier, V. K. Dhir and L. A. Momoda, 2002, "Heat transfer and pressure drop in narrow rectangular channels," Experimental Thermal Fluid Science, 26, pp. 53-64.

[53] W. Yu, D. M. France, M. W. Wambsganss and J. R. Hull, 2002, “Two-phase pressure drop, boiling heat transfer, and critical heat flux to water in a smalldiameter horizontal tube," Int. J. Multiphase Flow, 28, pp. 927-941.

[54] L. Zhang, J. Koo, L. Jiang, M. Asheghi, K. E. Goodson, J. G. Santiago and T. W. Kenny, 2002, "Measurements and modeling of two-phase flow in microchannels with nearly constant heat flux boundary conditions," J. Microelectromechanical Systems, 11 (1), pp. 12-19.

[55] D. Faulkner, M. Khotan and R. Shekarriz, 2003, "Practical design of a 1000 W/cm² cooling system," in proceedings of 19th IEEE Semi-Therm Symposium, pp. 223-230.

[56] W. Qu and I. Mudawar, 2003, "Flow boiling heat transfer in two-phase microchannel heat sinks - I. Experimental investigation and assessment of correlation methods," Int. J. Heat Mass Transfer, 46, pp. 2755-2771.

[57] W. Qu and I. Mudawar, 2003, "Flow boiling heat transfer in two-phase microchannel heat sinks - II. Annular two-phase flow model," Int. J. Heat Mass Transfer, 46, pp. 2773-2784. 
[58] B. Sumith, F. Kaminaga and K. Matsumura, 2003,"'Saturated flow boiling of water in a vertical small diameter tube," Experimental Thermal and Fluid Science, 27, pp 789-801.

[59] L. A. Campbell and S. Kandlikar, 2004, "Experimental study of heat transfer, pressure drop, and dryout for flow boiling of water in an oil heated minichannel," in proceedings of $2^{\text {nd }}$ Int. Conf. on Microchannels and Minichannels 2004.

[60] T. Henning, J. J. Brandner and K. Schubert, 2004, "Characterization of electrically powered micro-heat exchangers," Chemical Engineering J., 101, pp. 339-345.

[61] W. Qu and I. Mudawar, 2004, "Transport phenomena in two-phase micro-channel heat sinks," J. Electronics Packaging, 126, pp. 213-224.

[62] J. R. Thome, V. Dupont and A. M. Jacobi, 2004, "Heat transfer model for evaporation in microchannels. Part I: presentation of the model," Int. J. Heat Mass Transfer, 47, pp. 3375-3385.

[63] J. R. Thome, V. Dupont and A. M. Jacobi, 2004, "Heat transfer model for evaporation in microchannels. Part II: comparison with the database,” Int. J. Heat Mass Transfer, 47, pp. 3387-3401.

[64] W. Zhang, T. Hibiki and K. Mishima, 2004, "Correlation for flow boiling heat transfer in mini-channels," Int. J. Heat Mass Transfer, 47, pp. 5749-5763.

[65] J. Lee and I. Mudawar, 2005, "Two-phase flow in high-heat flux micro-channel heat sink for refrigeration cooling applications: Part II - heat transfer characteristics," Int. J. Heat Mass Transfer, 48, pp. 941-955.

[66] Y. M. Lie and T. F. Lin, 2005, "Saturated flow boiling heat transfer and associated bubble characteristics of R-134a in a narrow annular duct," Int. J. Heat Mass Transfer, 48, pp. 5602-5615.

[67] D. Liu, P. Lee and S. V. Garimella, 2005, "Prediction of the onset of nucleate boiling in microchannel flow," Int. J. Heat Mass Transfer, 48, pp. 5134-4149.

[68] W. Zhang, T. Hibiki and K. Mishima, 2005, "Correlation for flow boiling heat transfer at low liquid Reynolds number in small diameter channels," J. Heat Transfer, 127, pp. $1214-1221$.

[69] T. Chen and S. V. Garimella, 2006, "Measurements and high-speed visualizations of flow boiling of a dielectric fluid in a silicon microchannel heat sink," Int. J. Multiphase Flow, 32, pp. 957-971. 
[70] T. Chen and S. V. Garimella, 2006, "Effects of dissolved air on subcooled flow boiling of a dielectric coolant in a microchannel heat sink," ASME J. Electronic Packaging, 128 (4), pp. 398-404.

[71] T. Yen, M. Shoji, F. Takemura, Y. Suzuki and N. Kasagi, 2006, "Visualization of convective boiling heat transfer in single microchannels with different shaped cross-sections," Int. J. Heat Mass Transfer, 49, pp. 3884-3894.

[72] J. P. Calame, R. E. Myers, S. C. Binari, F. N. Wood and M. Garven, 2007, "Experimental investigation of microchannel coolers for the high heat flux thermal management of GaN-on-SiC semiconductor devices," Int. J. Heat Mass Transfer, 50, pp. 4767-4779.

[73] T. Chen and S. V. Garimella, 2007, "Flow boiling heat transfer to a dielectric coolant in a microchannel heat sink," IEEE Transactions of Components and Packaging Technologies, 30 (1), pp. 24 - 31.

[74] T. Harirchian and S. V. Garimella, "Microchannel size effects on local flow boiling heat transfer to a dielectric fluid," Int. J. Heat Mass Transfer (available online).

[75] C. Huh and M. H. Kim, 2007, "Pressure drop, boiling heat transfer and flow patterns during flow boiling in a single microchannel," Heat Transfer Engineering, 28 (8-9), pp. 730-737.

[76] V. V. Kuznetsov and A. S. Shamirzaev, 2007, "Boiling heat transfer for Freon R21 in rectangular minichannel," Heat Transfer Engineering, 28 (8-9), pp. 738745.

[77] D. Liu and S. V. Garimella, 2007, "Flow boiling heat transfer in microchannels," J. Heat Transfer, 129 (10), pp 1321-1332.

[78] J. V. S. Peters and S. G. Kandlikar, 2007, "Further evaluation of a flow boiling correlation for microchannels and minichannels," in proceeding of $5^{\text {th }}$ Int. Conf. on Nanochannels, Microchannels and Minichannels, ICNMM207-30027.

[79] S. L. Qi, P. Zhang, R. Z. Wang and L. X. Xu, 2007, "Flow boiling of liquid nitrogen in micro-tubes: Part II - Heat transfer characteristics and critical heat flux,” Int. J. Heat Mass Transfer, 50, pp. 5017-5030.

[80] G. Ribatski, W. Zhang, L. Consolini, J. Xu and J. R. Thome, 2007, "On the prediction of heat transfer in micro-scale flow boiling," Heat transfer Engineering, 28 (10), pp. 842-851. 
[81] J. L. Xu, S. Shen, Y. H. Gan, Y. X. Li, W. Zhang, and Q. C. Su, 2005, “Transient flow pattern based microscale boiling heat transfer mechanism," J.

Micromechanics Microengineering, 15, 1344-1361.

[82] S. Saitoh, H. Daiguji and E. Hihara, 2007, "Correlation for boiling heat transfer of R-134a in horizontal tubes including effect of tube diameter," Int. J. Heat Mass Transfer, 50, pp. 5215-5225.

[83] B. Schneider, A. Kosar and Y. Peles, 2007, "Hydrodynamic cavitation and boiling in refrigerant (R-123) flow inside microchannels," Int. J. Heat Mass Transfer, 50, pp. 2838-2854.

[84] H. Wang, S. V. Garimella and J. Y. Murthy, 2007, "Characteristics of an evaporating thin film in a microchannel," Int. J. Heat Mass Transfer, 50, pp. 3933-3942.

[85] P. S. Lee and S. V. Garimella, 2008, "Saturated flow boiling heat transfer and pressure drop in silicon microchannel arrays," Int. J. Heat Mass Transfer, 51 (34), pp 789-806.

[86] M. B. Bowers and I. Mudawar, 1994, "Two-phase electronic cooling using minichannel and micro-channel heat sinks: Part2 - Flow rate and pressure drop constraints," J. Electronic Packaging, 116, pp. 298-304.

[87] K. A. Triplett, S. M. Ghiaasiaan, S. I. Abdel-Khalik, A. LeMouel and B. N. McCord, 1999, "Gas-liquid two-phase flow in microchannels. Part II: Void fraction and pressure drop," Int. J. Multiphase Flow, 25, pp. 395-410.

[88] T. N. Tran, M. C. Chyu, M. W. Wambsganss and D. M. France, 2000, "Twophase pressure drop of refrigerants during flow boiling in small channels: an experimental investigation and correlation development," Int. J. Multiphase Flow, 26, pp. 1739-1754.

[89] J. Lee and I. Mudawar, 2005, "Two-phase flow in high-heat flux micro-channel heat sink for refrigeration cooling applications: Part I - pressure drop characteristics," Int. J. Heat Mass Transfer, 48, pp. 928-940.

[90] J. Lee and I. Mudawar, 2006, "Implementation of microchannel evaporator for high-heat-flux refrigeration cooling applications," J. Electronic Packaging, 128, pp. 30-37.

[91] Y. K. Hwang and M. S. Kim, 2006, "The pressure drop in microtubes and the correlation development," Int. J. Heat Mass Transfer, 49, pp. 1804-1812. 
[92] M. B. Bowers and I. Mudawar, 1994, "High flux boiling in low flow rate, low pressure drop mini-channel and micro-channel heat sinks," Int. J. Heat Mass Transfer, 37, pp. 321-332.M

[93] M. B. Bowers and I. Mudawar, 1994, "Two-phase electronic cooling using minichannel and micro-channel heat sinks: Part1 - Design criteria and heat diffusion constraints," J. Electronic Packaging, 116, pp. 290-297.

[94] G. P. Celata, M. Cumo and A. Mariani, 1994, "Burnout in Highly Subcooled Water Flow Boiling in Small Diameter Tubes,” Int. J. Heat Mass Transfer, 36, pp. 1269-1285.

[95] G. M. Roach Jr., S. I. Abdel-Khalik, S. M. Ghiaasiaan, M. F. Dowling and S. M. Jeter, 1999, "Low-flow critical heat flux in heated microchannels," Nuclear Science and Engineering, 131, pp. 411-425.

[96] J. C. Sturgis and I. Mudawar, 1999, "Critical heat flux in a long, rectangular channel subjected to one-sided heating - I. Flow visualization," Int. J. Heat Mass Transfer, 42, pp. 1835-1847.

[97] J. C. Sturgis and I. Mudawar, 1999, "Critical heat flux in a long, rectangular channel subjected to one-sided heating - II. Analysis of critical heat flux data," Int. J. Heat Mass Transfer, 42, pp. 1849-1862.

[98] A. Kosar and Y. Peles, 2007, "Critical heat flux of R-123 in silicon-based microchannels," J. Heat Transfer, 129 (7), pp. 884-851.

[99] R. Revellin and J.R. Thome, 2008, "A theoretical model for the prediction of the critical heat flux in heated microchannels," Int. J. Heat Mass Transfer, 51 (5-6), pp. 1216-1225.

[100] J. M. Ma and G. P. Peterson, 1996, "The interline heat transfer of evaporating thin films along a micro grooved surface," J. of Heat Transfer, 118, pp. 747-755.

[101] J. M. Ha and G. P. Peterson, 1998, "Capillary performance of evaporating flow in microgrooves - an analytical approach for very small tilt angles," J. of Heat Transfer, 120, pp. 452-457.

[102] K. A. Triplett, S. M. Ghiaasiaan, S. I. Abdel-Khalik and D. L. Sadowski, 1999, "Gas-liquid two-phase flow in microchannels. Part I: Two-phase flow patterns," Int. J. Multiphase Flow, 25, pp. 377-394.

[103] L. Jiang and M. Wong, 2001, "Forced convection boiling in a microchannel heat sink," J. Microelectromechanical Systems, 10 (1), pp. 80-87. 
[104] V. G. Nino, P. S. Hrnjak and T. A. Newell, 2003, “Two-phase flow visualization of R-134a in a multiport microchannel tube," Heat Transfer Engineering, 24 (1), pp. 41-52.

[105] X. Tu and P. S. Hrnjak, 2003, "Pressure drop and visualization of R-134a twophase flow in a rectangular microchannel," ASHRAE Transactions, 109 (1), CH03-13-4.

[106] D. K. Hollingsworth, 2004, "Liquid crystal imaging of flow boiling in minichannels," in proceedings of $2^{\text {nd }}$ Int. Conf. on Microchannels and Minichannels 2004.

[107] M. Molki, P. Mahendra and V. Vengala, 2004, "Visualization and modeling of flow boiling of R-134a in minichannels with transverse ribs," Heat Transfer Engineering, 25, pp. 94-103.

[108] P. Balasubramanian and S. G. Kandlikar, 2005, "Experimental study of flow patterns, pressure drop, and flow instabilities in parallel rectangular minichannels," Heat Transfer Engineering, 26 (3), pp. 20-27.

[109] A. Morgante and J. Fabre, 2005, "Two-phase flow in square mini-channels: Flow pattern transition," in proceedings of $3^{\text {rd }}$ Int. Conf. on Microchannels and Minichannels, pp. 691-695.

[110] Y. M. Lie and T. F. Lin, 2006, "Subcooled flow boiling heat transfer and associated bubble characteristics of R-134a in a narrow annular duct," Int. J. Heat Mass Transfer, 49, pp. 2077-2089.

[111] R. Revellin, V. Dupont, T. Ursenbacher, J.R. Thome and I. Zun, 2006, "Characterization of diabatic two-phase flows in microchannels: Flow parameter results for R-134a in a 0.5 mm channel,” Int. J. Multiphase Flow, 32, pp. 755-774.

[112] K. H. Chang and C. Pan, 2007, "Two-phase flow instability for boiling in a microchannel heat sink," Int. J. Heat Mass Transfer, 50, pp. 2078-2088.

[113] S. Hardt, B. Schilder, D. Tiemann, G. Kolb, V. Hessel and P. Stephan, 2007, "Analysis of flow patterns emerging during evaporation in parallel microchannels, “ Int. J. Heat Mass Transfer, 50, pp. 226-239.

[114] T. Hibiki, T. Hazuku, T. Takamasa and M. Ishii, 2007, "Some characteristics of developing bubbly flow in a vertical mini pipe,’ Int. J. Heat Fluid Flow, 28, pp. 1034-1048. 
[115] C. Huh, J. Kim and M. H. Kim, 2007, "Flow pattern transition instability during flow boiling in a single microchannel," Int. J. Heat Mass Transfer, 50, pp. 10491060.

[116] W. K. Kuan and S. G. Kandlikar, 2007, "Experimental study on the effect of stabilization on flow boiling heat transfer in microchannels," Heat Transfer Engineering, 28 (8-9), pp. 746-756.

[117] C.-J. Kuo and Y. Peles, 2007, "Local measurement of flow boiling in structured surface microchannels," Int. J. Heat Mass Transfer, 50, pp. 4513-4526.

[118] S.L. Qi, P. Zhang, R. Z. Wang, L. X. Xu, 2007, "Flow boiling of liquid nitrogen in micro-tubes: Part I - The onset of nucleate boiling, two-phase flow instability and two-phase flow pressure drop," Int. J. Heat Mass Transfer, 50, pp. 4999-5016.

[119] R. Revellin and J.R. Thome, 2007, "Experimental investigation of R-134a and R245 fa two-phase flow in microchannels for different flow conditions," Int. J. Heat Fluid Flow, 28, pp. 63-71.

[120] G. Wang, P. Cheng and H. Wu, 2007, "Unstable and stable flow boiling in parallel microchannels and in a single microchannel," Int. J. Heat Mass Transfer, 50, pp. 4297-4310.

[121] B. Alm, U. Imke, R. Knitter, U. Schygulla and S. Zimmermann, 2008, "Testing and simulation of ceramic micro heat exchangers," Chemical Engineering Journal, 135, pp. 179-184.

[122] R. Revellin, B. Agostini, T. Ursenbacher and J. R. Thome, 2008, "Experimental investigation of velocity and length of elongated bubbles for flow of R-134a in a $0.5 \mathrm{~mm}$ microchannel," Experimental Thermal and Fluid Science, 32, pp. 870881 .

[123] G. Wang, P. Cheng and A.E. Bergles, 2008, "Effects of inlet/outlet configurations on flow boiling instability in parallel microchannels," Int. J. Heat Mass Transfer, in press.

[124] S. Edelstein, A. J. Perez and J. C. Chen, 1984, "Analytic representation of convective boiling functions," American Institute of Chemical Engineers Journal, 30 (5), pp. 840-841.

[125] J. C. Chen, 1966, "Correlation for boiling heat transfer to saturated fluids in convective flow," I\&EC Process Design and Development, 5 (3), pp. 322-329. 
[126] D. L. Bennett and J. C. Chen, 1980, "Forced convective boiling in vertical tubes for saturated pure components and binary mixtures," American Institute of Chemical Engineers Journal, 26 (3), pp. 454-461.

[127] D.L. Bennett, M.W. Davies, and B.L. Hertzler, 1980, "The suppression of saturated nucleate boiling by forced convective flow," American Institute of Chemical Engineers Symposium Series, 76 (199), pp. 91-103.

[128] M. M. Shah, 1982, "Chart correlation for saturated boiling heat transfer: equations and further study," ASHRAE Transactions, 88, pp.185-196.

[129] K. E. Gungor and R. H. S. Winterton, 1986, "A general correlation for flow boiling in tubes and annuli," Int. J. Heat Mass Transfer, 29 (3), pp. 315-358.

[130] S. G. Kandlikar, 1990, "A general correlation for saturated two-phase flow boiling heat transfer inside horizontal and vertical tubes," J. Heat Transfer, 112, pp. 219-228.

[131] S. G. Kandlikar, 1991, "A model for correlating flow boiling heat transfer in augmented tubes and compact evaporators," J. Heat Transfer, 113, pp. 966-972.

[132] Z. Liu and R. H. S. Winterton, 1991, "A general correlation for saturated and subcooled flow boiling in tubes and annuli, based on a nucleate pool boiling equation," Int. J. Heat Mass Transfer, 34 (11), pp. 2759-2766.

[133] D. Steiner and J. Taborek, 1992, "Flow boiling heat transfer in vertical tubes correlated by an asymptotic model," Heat Transfer Engineering, 13 (2), pp. 43-68.

[134] B. S. Haynes and D. F. Fletcher, 2003, "Subcooled flow boiling heat transfer in narrow passages," Int. J. Heat Mass Transfer, 46, pp. 3673-3682.

[135] P. Balasubramanian and S. G. Kandlikar, 2004, "An extension of the flow boiling correlation to transition, laminar, and deep laminar flows in minichannel and microchannels," Heat Transfer Engineering, 25 (3), pp. 86-93.

[136] R. Yun, J. H. Heo and Y. Kim, 2007, "Erratum to "Evaporative heat transfer and pressure drop of R410A in microchannels", [Int. J. Refrigeration 29 (2006) 92100],’ Int. J. Refrigeration, 30, pp. 1468.

[137] M. G. Cooper, 1984, "Heat flow rates in saturated nucleate pool boiling - A wideranging examination using reduced properties," Advances in Heat Transfer, 16, pp. 157-239. 
[138] D. Gorenflo, 1993, "Pool boiling," VDI Heat Atlas, Verein Deutscher Ingenieure, VDI-Gesellschaft Verfahrenstechnik und Chemieingenieurwesen, ISBN3184009157.

[139] S. S. Bertsch, E. A. Groll, and S. V. Garimella, 2008, "A composite heat transfer correlation for saturated flow boiling in small channels," International Journal of Heat and Mass Transfer, (in review). 